\title{
Varieties of Graphoids and Birkoff's Theorem for Graphs
}

\author{
Symeon Bozapalidis ${ }^{a}$ and Antonios Kalampakas ${ }^{b}$
}

\begin{abstract}
The algebraic structure of graphoids is used in order to obtain the wellknown Birkhoff's theorem in the framework of graphs. Namely we establish a natural bijection between the class of $\Sigma$-graphoids and the class of strong congruences over $G R(\Sigma, X)$, which is the free graphoid over the doubly ranked alphabet $\Sigma$ and the set of variables $X$.
\end{abstract}

Keywords: hypergraphs, graphoids, graph congruences

\section{Introduction}

In theoretical computer science structural aspects such as syntax and semantics have been examined by methods of universal algebra. For example, in order to describe the semantics of program schemes, algebras can be used as models in which we suitably interpret all the involved syntactic symbols.

The well known Completeness Theorem (cf. [7]) states that an equation $t=t^{\prime}$, over a type $\Gamma$, is valid on the models of a set of equations $\mathcal{E}$, over $\Gamma$, if and only if we can go from $t$ to $t^{\prime}$ and vise versa through $\mathcal{E}$, this implies that semantics is equivalent to syntax. On the other hand every variety of algebras of type $\Gamma$ is equationally defined.

Our aim in the present paper is to obtain the above results within the framework of graphs. This will lead to the development of a robust graph rewriting theory analogous with the well established term rewriting theory (cf. [11], [3]). The graphs we consider have edges labeled over a doubly ranked alphabet $\Sigma$ and are equipped with a designated sequence of begin and end nodes. Moreover they can be combined in two basic ways: by horizontal composition and by disjoint union. The set $G R(\Sigma)$ of all such graphs is a graphoid, that is a magmoid (cf. [1, 2]) satisfying a finite set of standard equations (cf. [6]). Actually it is the free such structure over $\Sigma$ (cf.

\footnotetext{
${ }^{a}$ Department of Mathematics, Aristotle University of Thessaloniki, 54124, Thessaloniki, Greece E-mail: bozapali@math.auth.gr

${ }^{b}$ Department of Mathematics and Statistics, College of Engineering, American University of the Middle East, 15453, Egaila, Kuwait E-mail: antonios.kalampakas@aum.edu.kw
} 
[4]). This important result allows us to construct free objects within a variety of graphoids and it is a cornerstone in the present theory.

The main result of this paper is Birkhoff's Theorem for graphs stating that there is a bijection between the class of $\Sigma$ - $G$-varieties (i.e., varieties of $\Sigma$-designated graphoids) and the class of strong congruences over $G R(\Sigma \cup X)$, where $X$ a countable set of variables. This implies a Completeness Theorem for graphs: a graph equation $G=G^{\prime}$, of type $\Sigma$, is valid on the models of a set of graph equations $\mathcal{E}$, over $\Sigma$, iff we can go from $G$ to $G^{\prime}$ and vise versa through $\mathcal{E}$. Moreover every $\Sigma$ - $G$-variety is equationally defined.

The paper is divided into 7 sections. In Section 2 we define the categories of semi-magmoids and magmoids and we construct the free objects within these categories. Moreover, in the second subsection, we see how the set $G R(\Sigma)$ of all graphs over a finite doubly ranked alphabet $\Sigma$ can be structured into a magmoid. This magmoid is generated by the elements of $\Sigma$, viewed as graphs together with a finite set $\bar{D}$ of elementary graphs. Furthermore, we present an important theorem that provides a finite complete set of equations $(E)$, over $\Sigma \cup \bar{D}$, axiomatizing graphs in the sense that two graphs are isomorphic, if and only if, one can be transformed into the other by using these equations.

Graphoids are defined in the first part of Section 3, they are pairs $(M, D)$ consisting of a semi-magmoid $M$ and a set $D \subseteq M$ such that the equations $(E)$ are satisfied inside $M$. We prove that the set $G R(\Sigma)$ is actually the free graphoid generated by $\Sigma$. In addition we introduce $\Sigma$-graphoids which are graphoids $(M, D)$ equipped with a function interpreting the elements of $\Sigma$ by elements of $M$ of the same rank. In the second subsection, by virtue of $\Sigma$-graphoids, we introduce a substitution operation on graphs i.e., substitution of graphs inside graphs and more generally substitution of graphoid elements inside graphs. This operation is associative and allows us to explicitly describe the elements of the sub- $\Sigma$-graphoid generated by a set $A$. In the last subsection we see that the cartesian product (resp. directed union) of a family (resp. directed family) of semi-graphoids becomes a semi-graphoid in a natural way.

Congruences on $\Sigma$-graphoids are defined in Section 4 , the quotient of a $\Sigma$ graphoid by a congruence can be organized into a $\Sigma$-graphoid and congruences can be characterized by virtue of the substitution operation. The definition of strong congruences is obtained by suitably extending the notion of a congruence. Moreover, given a relation on the set of graphs we construct the smallest congruence (resp. strong congruence) containing this relation.

In the next section we introduce varieties of $\Sigma$-graphoids. A variety is class of $\Sigma$-graphoids closed under cartesian products, sub- $\Sigma$-graphoids and quotients. Any variety of graphoids is closed under directed union; additionally a $\Sigma$-graphoid belongs to a variety if and only if every finitely generated sub- $\Sigma$-graphoid of it belongs to the variety. In the same section we construct the free $\Sigma$-graphoid over a variety generated by a set $X$, its elements can be viewed as "graphs inside the variety".

In the sixth section we establish the main theorem of our paper. We say that a graph equation $\left(G, G^{\prime}\right)$ is satisfied by a $\Sigma$-graphoid (or that the $\Sigma$-graphoid is a 
model of this equation) whenever the equation holds for all possible assignments of its variables in that $\Sigma$-graphoid. We denote by $\operatorname{Mod}\left(G, G^{\prime}\right)$ the class of all models of $\left(G, G^{\prime}\right)$ and for a set of equations $\mathcal{E}$ we denote by $\operatorname{Mod}(\mathcal{E})$ the intersection of all the corresponding classes. This set actually constitutes a $\Sigma$ - $G$-variety and we say that a $\Sigma-G$-variety is equationally defined whenever it can be obtained in this way. In the opposite direction, given a class $\mathcal{K}$ of $\Sigma$-graphoids we denote by $E q(\mathcal{K})$ the set of all equations that are satisfied by all the elements of $\mathcal{K}$. It is proved that every $\Sigma$ - $G$-variety $\mathcal{V}$ is equationally defined, i.e., $\mathcal{V}=\operatorname{Mod}(E q(\mathcal{V}))$, moreover the variety generated by a class $\mathcal{K}$ of $\Sigma$-graphoids is equal with $\operatorname{Mod}(E q(\mathcal{K}))$. On the other hand, if $\mathcal{R}$ is a strong congruence on graphs then $E q(\operatorname{Mod}(\mathcal{R}))=\mathcal{R}$. By virtue of this result we prove (the graph analog of the well known Completeness Theorem) that if an equation is satisfied by every model of a set of equations $\mathcal{E}$ then we can go from $G$ to $G^{\prime}$ and vise versa. The Birkhoff's Theorem for graphs follows, namely we construct a bijection between the class of all $\Sigma$ - $G$-varieties and the class of all strong graph congruences over $\Sigma$. As an interesting application of this result we can associate to every graph language $L$ its syntactic variety $\mathcal{V}_{L}$, which is the variety corresponding to the syntactic strong congruence defined by $L$.

The relation between graph and pattern congruences is examined in Section 7. The notions of pattern substitution, pattern congruence (resp. strong pattern congruence) and quotient magmoid are presented and a characterization of pattern congruences (resp. strong pattern congruences) via substitution is given. A bijection between graph congruences (resp. strong graph congruences) and pattern congruences (resp. strong pattern congruences) containing $(E)$ is established. Furthermore we prove that the graph congruence which is generated by the projection on graphs of a pattern relation coincides with the projection on graphs of the pattern congruence that is generated by the same relation. A similar result is also proved for the inverse of this projection.

\section{Preliminaries}

\subsection{Semi-magmoids}

Recall that a doubly ranked alphabet is a set $A$ equipped with a function rank: $A \rightarrow \mathbb{N} \times \mathbb{N}$ (N the natural numbers). We write $A=\left(A_{m, n}\right)$ with

$$
A_{m, n}=\{a \mid a \in A, \operatorname{rank}(a)=(m, n)\},
$$

for all $m, n \in \mathbb{N}$.

A semi-magmoid is a doubly ranked set $M=\left(M_{m, n}\right)$ equipped with two operations

$$
\begin{gathered}
\circ: M_{m, n} \times M_{n, k} \rightarrow M_{m, k}, \quad m, n, k \geqslant 0 \\
\square: M_{m, n} \times M_{m^{\prime}, n^{\prime}} \rightarrow M_{m+m^{\prime}, n+n^{\prime}}, \quad m, n, m^{\prime}, n^{\prime} \geqslant 0
\end{gathered}
$$


which are associative in the obvious way and satisfy the distributivity law

$$
(f \circ g) \square\left(f^{\prime} \circ g^{\prime}\right)=\left(f \square f^{\prime}\right) \circ\left(g \square g^{\prime}\right)
$$

whenever all the above operations are defined.

A magmoid is a semi-magmoid $M=\left(M_{m, n}\right)$, equipped with a sequence of constants $e_{n} \in M_{n, n}(n \geqslant 0)$, called units, such that

$$
e_{m} \circ f=f=f \circ e_{n}, \quad e_{0} \square f=f=f \square e_{0}
$$

for all $f \in M_{m, n}$ and all $m, n \geqslant 0$, and the additional condition

$$
e_{m} \square e_{n}=e_{m+n}, \quad \text { for all } m, n \geqslant 0
$$

holds (cf. [1, 2]).

Notice that, due to the last equation, the element $e_{n}(n \geq 2)$ is uniquely determined by $e_{1}$. From now on $e_{1}$ will be simply denoted by $e$.

Subsemi-magmoids and morphisms of semi-magmoids (resp. magmoids) are defined in the obvious way.

Let $\Sigma$ be a doubly ranked alphabet. We denote by $S M(\Sigma)=\left(S M_{m, n}(\Sigma)\right)$ the smallest doubly ranked set satisfying the next items:

- $\Sigma_{m, n} \subseteq S M_{m, n}(\Sigma)$ for all $m, n \geq 0$,

- if $p \in S M_{m, n}(\Sigma)$ and $q \in S M_{n, k}(\Sigma)$ then their horizontal concatenation $p q \in$ $S M_{m, k}(\Sigma)$

- if $p \in S M_{m, n}(\Sigma)$ and $p^{\prime} \in S M_{m^{\prime}, n^{\prime}}(\Sigma)$ then their vertical concatenation $\underset{p^{\prime}}{p} \in$ $S M_{m+m^{\prime}, n+n^{\prime}}(\Sigma)$.

Let $\sim=\left(\sim_{m, n}\right)$ be the doubly ranked equivalence on $S M(\Sigma)$, compatible with horizontal and vertical concatenation, generated by the relations

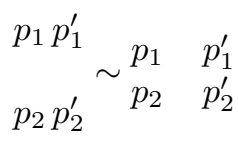

for all $p_{i}, p_{i}^{\prime}$ of suitable ranks. The quotient

$$
S M(\Sigma) / \sim=\left(S M_{m, n}(\Sigma) / \sim_{m, n}\right)
$$

is denoted $\operatorname{smag}(\Sigma)$ and is, by construction, a semi-magmoid. The elements of $\operatorname{smag}_{m, n}(\Sigma)$ are called $(m, n)$-patterns or patterns of rank $(m, n)$.

Convention. In order to avoid confusion in the pattern calculus instead of $\frac{p}{p^{\prime}}$ we write $\left(\begin{array}{c}p \\ p^{\prime}\end{array}\right)$. The associativity law takes the form

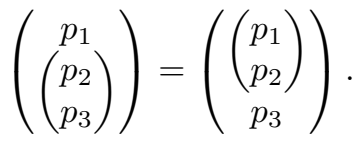


This common pattern will be denoted

$$
\left(\begin{array}{l}
p_{1} \\
p_{2} \\
p_{3}
\end{array}\right)
$$

The distributivity law takes the form

$$
\left(\begin{array}{l}
p_{1} p_{1}^{\prime} \\
p_{2}
\end{array} p_{2}^{\prime}\right)=\left(\begin{array}{l}
p_{1} \\
p_{2}
\end{array}\right)\left(\begin{array}{l}
p_{1}^{\prime} \\
p_{2}^{\prime}
\end{array}\right)
$$

Actually $\operatorname{smag}(\Sigma)$ is the free semi-magmoid generated by $\Sigma$ as confirms the next result.

Proposition 1. For every semi-magmoid $M=\left(M_{m, n}\right)$ and every doubly ranked function $f: \Sigma \rightarrow M$, there exists a unique morphism of semi-magmoids $\hat{f}$ : $\operatorname{smag}(\Sigma) \rightarrow M$ making the following triangle commutative.

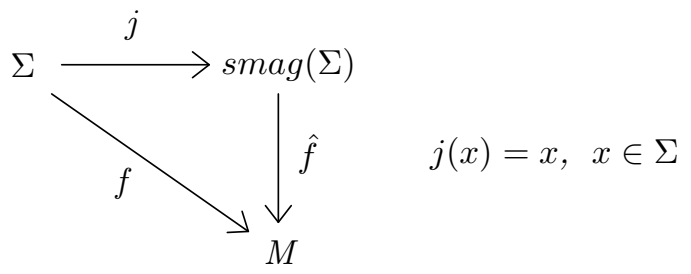

Actually, $\hat{f}$ is given by the clauses,

- $\hat{f}(x)=f(x)$, for all $x \in \Sigma$,

- $\hat{f}(p q)=\hat{f}(p) \circ \hat{f}(q), \quad \hat{f}\left(\begin{array}{c}p \\ p^{\prime}\end{array}\right)=\hat{f}(p) \square \hat{f}\left(p^{\prime}\right)$,

for all $p, q, p^{\prime} \in \operatorname{smag}(\Sigma)$ of suitable rank.

The construction of the free magmoid follows naturally. Let $\left(e_{n}\right)_{n \geq 0}$ be a sequence of symbols not in $\Sigma$ and denote by $\operatorname{mag}(\Sigma)$ the free semi-magmoid $\operatorname{smag}(\Sigma \cup$ $\left.\left\{e_{n} \mid n \geq 0\right\}\right)$ divided by the congruence generated by the relations

$$
e_{m} p \approx p \approx p e_{n}, \quad\left(\begin{array}{c}
e_{0} \\
p
\end{array}\right) \approx p \approx\left(\begin{array}{c}
p \\
e_{0}
\end{array}\right), \quad\left(\begin{array}{c}
e_{m} \\
e_{n}
\end{array}\right) \approx e_{m+n}
$$

for all $m, n \geq 0$ and all patterns $p$ of suitable rank. Then $\operatorname{mag}(\Sigma)$ clearly constitutes a magmoid which has a universal property analogous to the one stated in Proposition 1, i.e., $\operatorname{mag}(\Sigma)$ is the free magmoid generated by $\Sigma$ (cf. [4]).

\subsection{Graphs}

Now we introduce the magmoid of hypergraphs which will be of constant use throughout this paper. Given a finite alphabet $X$, we denote by $X^{*}$ the set of 
all words over $X$ and for every word $w \in X^{*},|w|$ denotes its length. Formally, a concrete $(m, n)$-graph over a doubly ranked alphabet $\Sigma=\left(\Sigma_{m, n}\right)$ is a tuple

$$
G=(V, E, s, t, l, \text { begin, end })
$$

where

- $V$ is the finite set of nodes,

- $E$ is the finite set of hyperedges,

- $s: E \rightarrow V^{*}$ is the source function,

- $t: E \rightarrow V^{*}$ is the target function,

- $l: E \rightarrow \Sigma$ is the labelling function such that $\operatorname{rank}(l(e))=(|s(e)|,|t(e)|)$ for every $e \in E$,

- begin $\in V^{*}$ with $\mid$ begin $\mid=m$ is the sequence of begin nodes and

- end $\in V^{*}$ with $|e n d|=n$ is the sequence of end nodes.

Notice that according to this definition vertices can be duplicated in the begin and end sequences of the graph and also at the sources and targets of an edge. For an edge $e$ of a hypergraph $G$ we simply write $\operatorname{rank}(e)$ to denote $\operatorname{rank}(l(e))$.

The specific sets $V$ and $E$ chosen to define a concrete graph $G$ are actually irrelevant. We shall not distinguish between two isomorphic graphs. Hence we have the following definition of an abstract graph. Two concrete $(m, n)$-graphs $G=(V, E, s, t, l$, begin, end $)$ and $G^{\prime}=\left(V^{\prime}, E^{\prime}, s^{\prime}, t^{\prime}, l^{\prime}\right.$, begin $^{\prime}$, end $\left.d^{\prime}\right)$ over $\Sigma$ are isomorphic iff there exist two bijections $h_{V}: V \rightarrow V^{\prime}$ and $h_{E}: E \rightarrow E^{\prime}$ commuting with source, target, labelling, begin and end in the usual way.

An abstract $(m, n)$-graph is defined to be the equivalence class of a concrete $(m, n)$-graph with respect to isomorphism. We denote by $G R_{m, n}(\Sigma)$ the set of all abstract $(m, n)$-graphs over $\Sigma$. Since we shall mainly be interested in abstract graphs we simply call them graphs except when it is necessary to emphasize that they are defined up to an isomorphism. Any graph $G \in G R_{m, n}(\Sigma)$ having no edges is called a discrete $(m, n)$-graph.

If $G$ is an $(m, n)$-graph represented by $(V, E, s, t, l$,begin,end $)$ and $H$ is an $(n, k)$-graph represented by $\left(V^{\prime}, E^{\prime}, s^{\prime}, t^{\prime}, l^{\prime}\right.$, begin $\left.{ }^{\prime}, e n d^{\prime}\right)$ then their product $G \circ H$ is the $(m, k)$-graph represented by the concrete graph obtained by taking the disjoint union of $G$ and $H$ and then identifying the $i$ th end node of $G$ with the $i$ th begin node of $H$, for every $i \in\{1, \ldots, n\}$; also, $\operatorname{begin}(G \circ H)=\operatorname{begin}(G)$ and $\operatorname{end}(G \circ H)=$ $\operatorname{end}(H)$.

The sum $G \square H$ of arbitrary graphs $G$ and $H$ is their disjoint union with their sequences of begin nodes concatenated and similarly for their end nodes.

For instance let $\Sigma=\{a, b, c\}$, with $\operatorname{rank}(a)=(2,1) \operatorname{rank}(b)=(1,1)$ and $\operatorname{rank}(c)=(1,2)$. In the following pictures, edges are represented by boxes, nodes by dots, and the sources and targets of an edge by directed lines that enter and 
leave the corresponding box, respectively. The order of the sources and targets of an edge is the vertical order of the directed lines as drawn in the pictures. We display two graphs $G \in G R_{3,4}(\Sigma)$ and $H \in G R_{4,2}(\Sigma)$, where the $i$ th begin node is indicated by $b_{i}$, and the $i$ th end node by $e_{i}$.

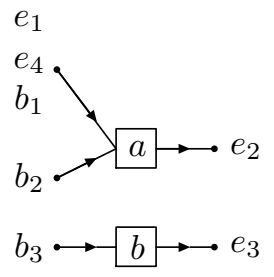

G

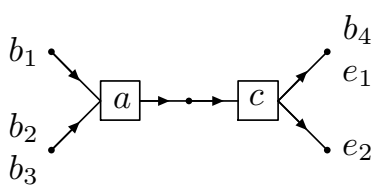

H

Then their product $G \circ H$ is the $(3,2)$-graph

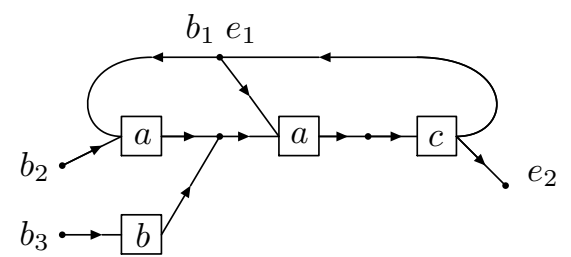

and, their sum $G \square H$ is the (7,6)-graph
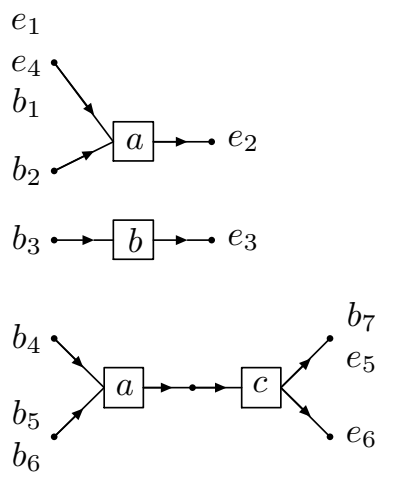

For every $n \in \mathbb{N}$ we denote by $E_{n}$ the discrete graph of rank $(n, n)$ with nodes $x_{1}, \ldots, x_{n}$ and $\operatorname{begin}\left(E_{n}\right)=\operatorname{end}\left(E_{n}\right)=x_{1} \cdots x_{n}$; we write $E$ for $E_{1}$. Note that $E_{0}$ is the empty graph.

It is straightforward to verify that $G R(\Sigma)=\left(G R_{m, n}(\Sigma)\right)$ with the operations defined above is a magmoid whose units are the graphs $E_{n}, n \geq 0$, see Lemma 6 of [10]. The discrete graphs of $G R(\Sigma)$ form obviously a sub-magmoid DISC of $G R(\Sigma)$ and the function sending each graph $G \in G R(\Sigma)$ to its underlying discrete graph is an epimorphism of magmoids

$$
\operatorname{disc}_{\Sigma}: G R(\Sigma) \rightarrow D I S C .
$$


Let us denote by $I_{p, q}$ the discrete $(p, q)$-graph having a single node $x$ and whose begin and end sequences are $x \cdots x$ ( $p$ times) and $x \cdots x$ ( $q$ times) respectively. Note that $I_{1,1}$ is equal with $E$. Let also $\Pi$ be the discrete $(2,2)$-graph having two nodes $x$ and $y$ and whose begin and end sequences are $x y$ and $y x$, respectively. Finally, for every $\sigma \in \Sigma_{m, n}$, we denote again by $\sigma$ the $(m, n)$-graph having only one edge and $m+n$ nodes $x_{1}, \ldots, x_{m}, y_{1}, \ldots, y_{n}$. The edge is labelled by $\sigma$, and the begin (resp. end sequence) of the graph is the sequence of sources (resp. targets) of the edge, viz. $x_{1} \cdots x_{m}$ (resp. $\left.y_{1} \cdots y_{n}\right)$.

As usual $\mathcal{S}_{m}$ stands for the group of all permutations of the set $\{1,2, \ldots, m\}$. Given a permutation $\alpha \in \mathcal{S}_{m}$

$$
\alpha=\left(\begin{array}{cccc}
1 & 2 & \ldots & m \\
\alpha(1) & \alpha(2) & \ldots & \alpha(m)
\end{array}\right)
$$

the discrete graph having $\left\{x_{1}, x_{2}, \ldots, x_{m}\right\}$ as set of nodes, $x_{\alpha(1)} x_{\alpha(2)} \cdots x_{\alpha(m)}$ as begin sequence and $x_{1} x_{2} \cdots x_{m}$ as end sequence, is denoted $\Pi_{\alpha}$ and is called the permutation graph associated with $\alpha$. Observe that $\Pi$ is the graph associated with the permutation

$$
\alpha=\left(\begin{array}{ll}
1 & 2 \\
2 & 1
\end{array}\right) .
$$

We denote by $\Pi_{n, 1}$ the graph associated with the permutation

$$
\left(\begin{array}{cccc}
1 & 2 & \cdots & n+1 \\
2 & \cdots & n+1 & 1
\end{array}\right)
$$

interchanging the last $n$ numbers with the first one.

For every $\sigma \in \Sigma_{m, n}, B F(\sigma)$ is the same as the graph $\sigma$, except that

$$
\operatorname{begin}(B F(\sigma))=\operatorname{begin}(\sigma) \operatorname{end}(\sigma)=x_{1} \cdots x_{m} y_{1} \cdots y_{n}
$$

and $\operatorname{end}(B F(\sigma))=\varepsilon$ (where $\varepsilon$ denotes the empty sequence).

Next important result provides a complete set of equations axiomatizing graphs.

Theorem 1 (cf. [4]). Let $\Sigma=\left\{\sigma_{1}, \ldots, \sigma_{r}\right\}$ and assume that in the graph $G \in$ $G R_{m, n}(\Sigma)$ the symbol $\sigma_{i}$ occurs $\lambda_{i}$ times, $(1 \leq i \leq r)$ then $G$ admits a decomposition of the form

$$
\begin{aligned}
& G=\Pi_{\alpha} \circ\left(I_{p_{1}, q_{1}} \square \cdots \square I_{p_{s}, q_{s}}\right) \circ \Pi_{\beta} \\
& \quad \circ\left(E_{n} \square B F\left(\sigma_{1}^{1}\right) \square \cdots \square B F\left(\sigma_{1}^{\lambda_{1}}\right) \square \cdots \square B F\left(\sigma_{r}^{1}\right) \square \cdots \square B F\left(\sigma_{r}^{\lambda_{r}}\right)\right)
\end{aligned}
$$

where $\alpha, \beta$ are suitable permutations and $\sigma_{i}^{1}=\cdots=\sigma_{i}^{\lambda_{i}}=\sigma_{i}, 1 \leq i \leq r$. Moreover, if

$$
\begin{aligned}
G^{\prime} & =\Pi_{\alpha^{\prime}} \circ\left(I_{p_{1}^{\prime}, q_{1}^{\prime}} \square \cdots \square I_{p_{t}^{\prime}, q_{t}^{\prime}}\right) \circ \Pi_{\beta^{\prime}} \\
& \circ\left(E_{n} \square B F\left(\sigma_{1}^{1}\right) \square \cdots \square B F\left(\sigma_{1}^{\lambda_{1}^{\prime}}\right) \square \cdots \square B F\left(\sigma_{r}^{1}\right) \square \cdots \square B F\left(\sigma_{r}^{\lambda_{r}^{\prime}}\right)\right)
\end{aligned}
$$


then $G=G^{\prime}$ if and only if we can transform $G$ into $G^{\prime}$ through the finite set of equations $(E)$ :

$$
\begin{gathered}
\Pi \circ \Pi=E \square E, \quad(E \square \Pi) \circ(\Pi \square E) \circ(E \square \Pi)=(\Pi \square E) \circ(E \square \Pi) \circ(\Pi \square E), \\
\left(E \square I_{2,1}\right) \circ I_{2,1}=\left(I_{2,1} \square E\right) \circ I_{2,1}, \quad\left(E \square I_{0,1}\right) \circ I_{2,1}=E, \quad \Pi \circ I_{2,1}=I_{2,1} \\
(\Pi \square E) \circ(E \square \Pi) \circ\left(I_{2,1} \square E\right)=\left(E \square I_{2,1}\right) \circ \Pi, \quad\left(E \square I_{0,1}\right) \circ \Pi=\left(I_{0,1} \square E\right), \\
I_{1,2} \circ\left(E \square I_{1,2}\right)=I_{1,2} \circ\left(I_{1,2} \square E\right), \quad I_{1,2} \circ\left(E \square I_{1,0}\right)=E, \quad I_{1,2} \circ \Pi=I_{1,2}, \\
I_{1,2} \circ I_{2,1}=E, \quad\left(I_{1,2} \square E\right) \circ(E \square \Pi) \circ(\Pi \square E)=\Pi \circ\left(E \square I_{1,2}\right), \\
\Pi \circ\left(E \square I_{1,0}\right)=\left(I_{1,0} \square E\right), \quad\left(I_{1,2} \square E\right) \circ\left(E \square I_{2,1}\right)=I_{2,1} \circ I_{1,2}, \\
\Pi_{p, 1} \circ(\sigma \square E)=(E \square \sigma) \circ \Pi_{q, 1}, \quad \text { where } \sigma \in \Sigma_{p, q}, \quad p, q \geq 0 .
\end{gathered}
$$

Now let us introduce the alphabet $\bar{D}$, formed by the following five symbols

$$
\bar{i}_{21}: 2 \rightarrow 1 \quad \bar{i}_{01}: 0 \rightarrow 1 \quad \bar{i}_{12}: 1 \rightarrow 2 \quad \bar{i}_{10}: 1 \rightarrow 0 \quad \bar{\pi}: 2 \rightarrow 2
$$

where $x: m \rightarrow n$ indicates that symbol $x$ has first rank $m$ and second rank $n$, and denote by $\operatorname{mag}(\Sigma \cup \bar{D})$ the free magmoid generated by the doubly ranked alphabet $\Sigma \cup \bar{D}$. We denote by

$$
\operatorname{val}_{\Sigma}: \operatorname{mag}(\Sigma \cup \bar{D}) \rightarrow G R(\Sigma)
$$

the unique magmoid morphism extending the function described by the assignments

$$
\begin{gathered}
\bar{i}_{21} \mapsto I_{2,1}, \quad \bar{i}_{01} \mapsto I_{0,1}, \quad \bar{i}_{12} \mapsto I_{1,2}, \quad \bar{i}_{10} \mapsto I_{1,0}, \quad \bar{\pi} \mapsto \Pi, \\
\sigma \mapsto \sigma, \quad \text { for all } \sigma \in \Sigma, \quad e_{n} \mapsto E_{n}, \text { for all } n \in \mathbb{N} .
\end{gathered}
$$

According to the previous theorem $v^{2} l_{\Sigma}$ is a surjection and $G R(\Sigma)$ is the quotient of $\operatorname{mag}(\Sigma \cup \bar{D})$ by the congruence generated by the relations $(E)$ :

$$
\begin{gathered}
\bar{\pi} \bar{\pi} \sim e_{2}, \quad\left(\begin{array}{c}
e \\
\bar{\pi}
\end{array}\right)\left(\begin{array}{c}
\bar{\pi} \\
e
\end{array}\right)\left(\begin{array}{c}
e \\
\bar{\pi}
\end{array}\right) \sim\left(\begin{array}{c}
\bar{\pi} \\
e
\end{array}\right)\left(\begin{array}{l}
e \\
\bar{\pi}
\end{array}\right)\left(\begin{array}{c}
\bar{\pi} \\
e
\end{array}\right), \\
\left(\begin{array}{c}
e \\
\bar{i}_{21}
\end{array}\right) \bar{i}_{21} \sim\left(\begin{array}{c}
\bar{i}_{21} \\
e
\end{array}\right) \bar{i}_{21}, \quad\left(\begin{array}{c}
e \\
\bar{i}_{01}
\end{array}\right) \bar{i}_{21} \sim e,
\end{gathered}
$$




$$
\begin{gathered}
\bar{\pi} \bar{i}_{21} \sim \bar{i}_{21}, \quad\left(\begin{array}{c}
e \\
\bar{i}_{01}
\end{array}\right) \bar{\pi} \sim\left(\begin{array}{c}
\bar{i}_{01} \\
e
\end{array}\right), \\
\left(\begin{array}{c}
\bar{\pi} \\
e
\end{array}\right)\left(\begin{array}{c}
e \\
\bar{\pi}
\end{array}\right)\left(\begin{array}{c}
\bar{i}_{21} \\
e
\end{array}\right) \sim\left(\begin{array}{c}
e \\
\bar{i}_{21}
\end{array}\right) \bar{\pi}, \\
\bar{i}_{12}\left(\begin{array}{c}
e \\
\bar{i}_{12}
\end{array}\right) \sim \bar{i}_{12}\left(\begin{array}{c}
\bar{i}_{12} \\
e
\end{array}\right), \quad \bar{i}_{12}\left(\begin{array}{c}
e \\
\bar{i}_{10}
\end{array}\right) \sim e, \\
\bar{i}_{12} \bar{\pi} \sim \bar{i}_{12}, \quad \bar{\pi}\left(\begin{array}{c}
e \\
\bar{i}_{10}
\end{array}\right) \sim\left(\begin{array}{c}
\bar{i}_{10} \\
e
\end{array}\right), \\
\left(\begin{array}{c}
\bar{i}_{12} \\
e
\end{array}\right)\left(\begin{array}{c}
e \\
\bar{\pi}
\end{array}\right)\left(\begin{array}{c}
\bar{\pi} \\
e
\end{array}\right) \sim \bar{\pi}\left(\begin{array}{c}
e \\
\bar{i}_{12}
\end{array}\right), \\
\bar{i}_{12} \bar{i}_{21} \sim e, \quad\left(\begin{array}{c}
\bar{i}_{12} \\
e
\end{array}\right)\left(\begin{array}{c}
e \\
\bar{i}_{21}
\end{array}\right) \sim \bar{i}_{21} \bar{i}_{12}, \\
\bar{\pi}_{m, 1}\left(\begin{array}{c}
\sigma \\
e
\end{array}\right) \sim\left(\begin{array}{c}
e \\
\sigma
\end{array}\right) \bar{\pi}_{n, 1},
\end{gathered}
$$

where $\sigma \in \Sigma_{m, n}, m, n \geq 0$ and $\bar{\pi}_{n, 1}$ is the pattern inductively defined by

$$
\bar{\pi}_{1,0}=e, \quad \bar{\pi}_{n, 1}=\left(\begin{array}{c}
\bar{\pi}_{n-1,1} \\
e
\end{array}\right)\left(\begin{array}{c}
e_{n-1} \\
\bar{\pi}
\end{array}\right)
$$

which will represent the graph $\Pi_{n, 1}$.

The next definition will be used later on. We call size of a pattern $p \in \operatorname{mag}(\Sigma \cup$ $\bar{D})$ the number of symbols of $\Sigma \cup \bar{D}$ occurring in $p$. The size of a graph $G \in G R(\Sigma)$ is then

$$
\operatorname{size}(G)=\min \left\{\operatorname{size}(p) \mid p \in \operatorname{val}_{\Sigma}^{-1}(G)\right\} .
$$

\section{Graphoids and their algebra}

\subsection{Free graphoids}

The algebraic structure underneath $G R(\Sigma)$ is that of a graphoid.

More precisely, a graphoid $\mathbf{M}=(M, D)$ consists of a semi-magmoid $M$ and a set

$$
D=\left\{e_{0}, e, \pi, i_{01}, i_{21}, i_{10}, i_{12}\right\},
$$

where $e_{0} \in M_{0,0}, e \in M_{1,1}, \pi \in M_{2,2}, i_{01} \in M_{0,1}, i_{21} \in M_{2,1}, i_{10} \in M_{1,0}, i_{12} \in M_{1,2}$ such that $\left(M, e_{0}, e\right)$ is a magmoid, i.e.,

$$
\text { (1) } e_{m} \circ f=f=f \circ e_{n}, \quad e_{0} \square f=f=f \square e_{0},
$$

where $e_{n}=e \square \cdots \square e$ ( $n$-times, $\left.n \geq 0\right), f \in M_{m, n},(m, n \geq 0)$, and additionally the following equations hold 
(2)

$$
\begin{gathered}
\pi \circ \pi=e_{2}, \quad(\pi \square e) \circ(e \square \pi) \circ(\pi \square e)=(e \square \pi) \circ(\pi \square e) \circ(e \square \pi) \\
(3) \quad\left(e \square i_{21}\right) \circ i_{21}=\left(i_{21} \square e\right) \circ i_{21}, \quad\left(e \square i_{01}\right) \circ i_{21}=e, \\
\pi \circ i_{21}=i_{21}, \quad\left(e \square i_{01}\right) \circ \pi=\left(i_{01} \square e\right), \\
(\pi \square e) \circ(e \square \pi) \circ\left(i_{21} \square e\right)=\left(e \square i_{21}\right) \circ \pi, \\
(4) \quad i_{12} \circ\left(e \square i_{12}\right)=i_{12} \circ\left(i_{12} \square e\right), \quad i_{12} \circ\left(e \square i_{10}\right)=e, \\
i_{12} \circ \pi=i_{12}, \quad \pi \circ\left(e \square i_{10}\right)=\left(i_{10} \square e\right), \\
\left(i_{12} \square e\right) \circ(e \square \pi) \circ(\pi \square e)=\pi \circ\left(e \square i_{12}\right), \\
(5) \quad i_{12} \circ i_{21}=e, \quad\left(i_{12} \square e\right) \circ\left(e \square i_{21}\right)=i_{21} \circ i_{12} . \\
\text { (6) } \quad \pi_{m, 1} \circ(f \square e)=(e \square f) \circ \pi_{n, 1}, \quad \text { for all } f \in M_{m, n},
\end{gathered}
$$

where the element $\pi_{m, 1} \in M_{m, 1}$ is defined by

$$
\pi_{1,0}=e, \quad \pi_{m, 1}=\left(\pi_{m-1,1} \square e\right) \circ\left(e_{m-1} \square \pi\right) .
$$

We point out that equation (6) holds in $G R(\Sigma)$ since it holds for all the letters of the alphabet $\Sigma$ (cf. [4]).

Observe that $\left(G R(\Sigma),\left\{E_{0}, E, \Pi, I_{0,1}, I_{2,1}, I_{1,0}, I_{1,2}\right\}\right)$ is a graphoid which from now on will be simply denoted by $G R(\Sigma)$.

Given graphoids $(M, D)$ and $\left(M^{\prime}, D^{\prime}\right)$, a semi-magmoid morphism $H: M \rightarrow M^{\prime}$ preserving $D$-sets, i.e., $H\left(e_{0}\right)=e_{0}^{\prime}, H(e)=e^{\prime}, H(\pi)=\pi^{\prime}$ and $H\left(i_{\kappa \lambda}\right)=i_{\kappa \lambda}^{\prime}$, is called a morphism of graphoids.

We have already discussed how the set $G R(\Sigma)$ can be structured into a graphoid; in fact it is the free graphoid generated by $\Sigma$.

Theorem 2. The doubly ranked function $j: \Sigma \rightarrow G R(\Sigma)$, with $j(\sigma)=\sigma$, for all $\sigma \in \Sigma$, has the following universal property: for any graphoid $\mathbb{M}=(M, D)$, $D=\left\{e_{0}, e, \pi, i_{10}, i_{12}, i_{01}, i_{21}\right\}$ and any doubly ranked function $f: \Sigma \rightarrow M$, there exists a unique morphism of graphoids $\bar{f}: G R(\Sigma) \rightarrow \mathbb{M}$ making commutative the following triangle.

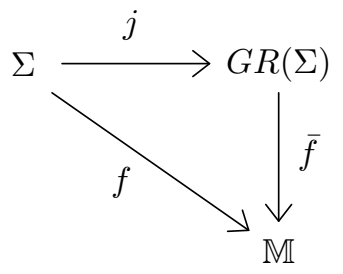

The morphism $\bar{f}$ is defined by the clauses 
- $\bar{f}(\sigma)=f(\sigma), \sigma \in \Sigma$,

- $\bar{f}\left(E_{0}\right)=e_{0}, \bar{f}(E)=e, \bar{f}(\Pi)=\pi, \bar{f}\left(I_{p, q}\right)=i_{p q}$,

- $\bar{f}\left(G_{1} \circ G_{2}\right)=\bar{f}\left(G_{1}\right) \circ \bar{f}\left(G_{2}\right)$,

- $\bar{f}\left(G_{1} \square G_{2}\right)=\bar{f}\left(G_{1}\right) \square \bar{f}\left(G_{2}\right)$,

for all graphs $G_{1}, G_{2}$ of suitable rank.

Proof. Since $\operatorname{mag}(\Sigma \cup \bar{D})$ is the free magmoid over $\Sigma \cup \bar{D}, f$ is uniquely extended into a morphism of magmoids $\hat{f}: \operatorname{mag}(\Sigma \cup \bar{D}) \rightarrow \mathbb{M}$ making commutative the triangle:

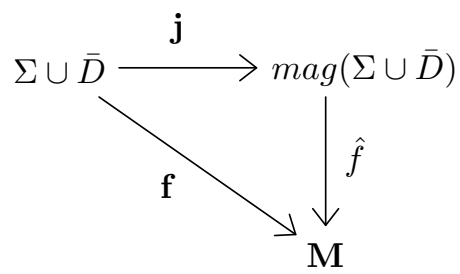

where

- $\mathbf{j}(\sigma)=j(\sigma), \sigma \in \Sigma$ and $\mathbf{j}(\alpha)=\alpha, \alpha \in \bar{D}$

- $\mathbf{f}(\sigma)=f(\sigma), \sigma \in \Sigma$ and $\mathbf{f}\left(\bar{i}_{21}\right)=i_{21}, \mathbf{f}\left(\bar{i}_{01}\right)=i_{01}, \mathbf{f}\left(\bar{i}_{12}\right)=i_{12}, \mathbf{f}\left(\bar{i}_{10}\right)=i_{10}$, $\mathbf{f}(\bar{\pi})=\pi$.

Since all the relations $(E)$ are valid in $\mathbf{M}$, the kernel of $\hat{f}$ includes the congruence ${ }_{(E)}$ generated by $(E)$, and thus $\hat{f}$ induces a unique graphoid morphism

$$
\bar{f}: \operatorname{mag}(\Sigma \cup \bar{D}) /=_{(E)} \longrightarrow \mathbf{M}
$$

rendering commutative the triangle

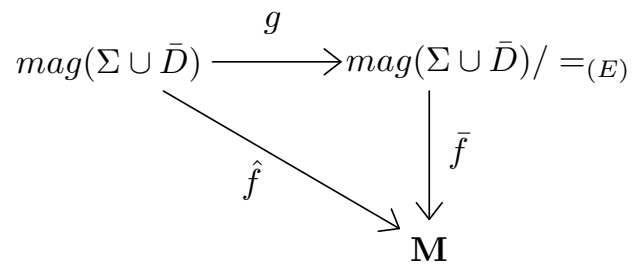

where $g$ is the canonical projection sending every element of $\operatorname{mag}(\Sigma \cup \bar{D})$ to its class with respect to $=_{(E)}$. The result comes by combining the above two diagrams and Theorem 1.

A graph homomorphism $H: G R(\Sigma) \rightarrow G R\left(\Sigma^{\prime}\right)$ is just a morphism of graphoids. Hence, by virtue of the previous theorem it is completely determined by its values $H(\sigma), \sigma \in \Sigma$. A graph homomorphism $H: G R(\Sigma) \rightarrow G R\left(\Sigma^{\prime}\right)$ is called a projection whenever $H(\Sigma) \subseteq \Sigma^{\prime}$. 
In the sequel, we mostly deal with $\Sigma$-graphoids, ( $\Sigma$ doubly ranked alphabet) which are graphoids $(M, D)$ equipped with a function $\mu: \Sigma \rightarrow M$ interpreting the letters $\sigma \in \Sigma$ by elements of $M$ with the same rank.

By virtue of Theorem 2 any graph $G$ of $G R(\Sigma)$ is interpreted, in a $\Sigma$-graphoid $\mathbf{M}=(M, D, \mu)$, by the element $\bar{\mu}(G)$. In other words, we are able to make graph theory inside any $\Sigma$-graphoid.

The set $G R(\Sigma)$ is a $\Sigma$-graphoid where $t$ is the function sending every element $\sigma \in \Sigma$ to the graph it represents.

If $\mathbf{M}=(M, D, \mu)$ and $\mathbf{M}^{\prime}=\left(M^{\prime}, D^{\prime}, \mu^{\prime}\right)$ are two $\Sigma$-graphoids then any graphoid morphism $H:(M, D) \rightarrow\left(M^{\prime}, D^{\prime}\right)$ commuting with the $\mu$-assignments, i.e., rendering commutative the triangle

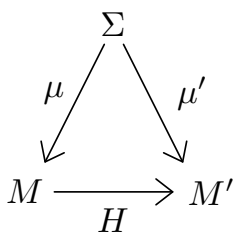

is called a $\Sigma$-graphoid morphism.

Graphs whose labels of edges are variables will be frequently used. From now on $X_{p}=\left\{x_{1}, \ldots, x_{p}\right\}$ is a set of doubly ranked variables with $\operatorname{rank}\left(x_{i}\right)=\left(\alpha_{i}, \beta_{i}\right)$, $1 \leq i \leq p$.

Convention. The $\Sigma$-graphoid $G R\left(\Sigma \cup X_{p}\right)$ will be denoted by $G R\left(\Sigma, X_{p}\right)$.

Theorem 3. The $\Sigma$-graphoid $G R(\Sigma, X)$ is free over $X$, i.e., for any $\Sigma$-graphoid $\mathbf{M}=(M, D, \mu)$ and any function $f: X \rightarrow M$ there is a unique $\Sigma$-graphoid morphism $\bar{f}: G R(\Sigma, X) \rightarrow \mathbf{M}$ such that the next diagram commutes

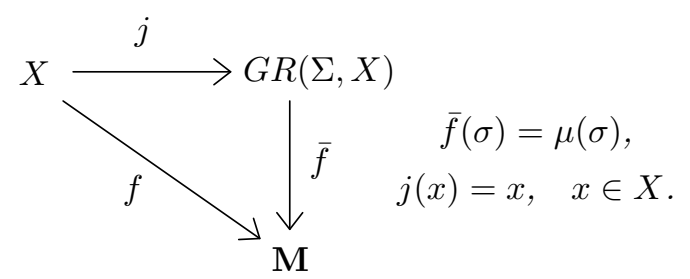

\subsection{The substitution operation}

The well known edge replacement operation on graphs (cf. [9], [8] ) can be defined with the help of Theorem 3 in an elegant way. Let $X=\left\{x_{1}, x_{2}, \ldots\right\}$ with $\operatorname{rank}\left(x_{i}\right)=\left(\alpha_{i}, \beta_{i}\right), i=1,2, \ldots$ and $X_{p}=\left\{x_{1}, \ldots, x_{p}\right\}$. For

$$
G_{i} \in G R_{\alpha_{i}, \beta_{i}}\left(\Sigma, X_{p}\right) \text {. }
$$

The function

$$
f: X_{p} \rightarrow G R\left(\Sigma, X_{k}\right), \quad f\left(x_{i}\right)=G_{i}
$$


is then uniquely extended into a $\Sigma$-graphoid morphism

$$
\bar{f}: G R\left(\Sigma, X_{p}\right) \rightarrow G R\left(\Sigma, X_{k}\right) .
$$

For all $G \in G R_{\alpha, \beta}\left(\Sigma, X_{p}\right)$, the graph $\bar{f}(G)$ is denoted by $G\left[G_{1}, \ldots, G_{p}\right]$ (it is the graph obtained by simultaneously replacing $x_{i}$ by $G_{i}$ inside $\left.G, i=1, \ldots, p\right)$.

Proposition 2. It holds

$$
G\left[G_{1}, \ldots, G_{p}\right]\left[G_{1}^{\prime}, \ldots, G_{k}^{\prime}\right]=G\left[G_{1}\left[G_{1}^{\prime}, \ldots, G_{k}^{\prime}\right], \ldots, G_{p}\left[G_{1}^{\prime}, \ldots, G_{k}^{\prime}\right]\right]
$$

for all graphs of suitable rank.

Proof. We define the functions

$$
f: X_{p} \rightarrow G R\left(\Sigma, X_{k}\right), \quad g: X_{k} \rightarrow G R\left(\Sigma, X_{s}\right)
$$

by setting

$$
f\left(x_{i}\right)=G_{i}(1 \leq i \leq p), \quad g\left(x_{j}\right)=G_{j}^{\prime}(1 \leq j \leq k),
$$

respectively. For uniqueness reasons invoked by Theorem 3 we get the equality

$$
\overline{\bar{g} \circ f}=\bar{g} \circ \bar{f} .
$$

Since $(\bar{g} \circ f)\left(x_{i}\right)=\bar{g}\left(f\left(x_{i}\right)\right)=\bar{g}\left(G_{i}\right)=G_{i}\left[G_{1}^{\prime}, \ldots, G_{k}^{\prime}\right]$ we get

$$
\begin{aligned}
G\left[G_{1}, \ldots, G_{p}\right]\left[G_{1}^{\prime}, \ldots, G_{k}^{\prime}\right] & =\bar{g}\left(G\left[G_{1}, \ldots, G_{p}\right]\right) \\
& =\bar{g}(\bar{f}(G))=(\bar{g} \circ \bar{f})(G)=\overline{\bar{g} \circ f}(G) \\
& =G\left[G_{1}\left[G_{1}^{\prime}, \ldots, G_{k}^{\prime}\right], \ldots, G_{p}\left[G_{1}^{\prime}, \ldots, G_{k}^{\prime}\right]\right]
\end{aligned}
$$

as wanted.

Let $\xi_{m, n}$ be a new symbol with rank $(m, n)$ and denote by $F R_{m, n}^{\alpha, \beta}(\Sigma, X)$ the subset of $G R_{\alpha, \beta}\left(\Sigma, X \cup\left\{\xi_{m, n}\right\}\right)$ with just one occurrence of $\xi_{m, n}$; its elements are called frames with exterior $\operatorname{rank}(\alpha, \beta)$ and interior $\operatorname{rank}(m, n)$. The set $F R_{m, n}^{\alpha, \beta}(\Sigma, X)$ acts on $G R_{m, n}(\Sigma, X)$ via substitution at $\xi_{m, n}$ : for $F \in F R_{m, n}^{\alpha, \beta}(\Sigma, X)$ and $G \in G R_{m, n}(\Sigma, X)$,

$$
F \cdot G=F\left[G / \xi_{m, n}\right] .
$$

The substitution of graphs inside graphs can be extended into a substitution of graphoid elements inside graphs. This will be achieved be means of the universal property described in Theorem 3. Let $a_{1}, \ldots, a_{p}$ be elements of a $\Sigma$-graphoid $\mathbf{M}=$ $(M, D, \mu)$

$$
a_{i} \in M_{\alpha_{i}, \beta_{i}}, \quad i=1, \ldots, p
$$

and consider the $\Sigma$-graphoid morphism $\bar{h}: G R\left(\Sigma, X_{p}\right) \rightarrow \mathbf{M}$ determined by the assignments

$$
h\left(x_{1}\right)=a_{1}, \ldots, h\left(x_{p}\right)=a_{p} .
$$

For any graph $G \in G R\left(\Sigma, X_{p}\right)$ we denote by $G\left[a_{1}, \ldots, a_{p}\right]$ the element $\bar{h}(G)$. Notice that from Theorem 3 it holds $\bar{h}(\sigma)=\mu(\sigma)$. The so defined mixed substitution operation has the nice properties of graph substitution. More precisely, 
Proposition 3 (Associativity law for mixed substitution). It holds

$$
G\left[G_{1}, \ldots, G_{p}\right]\left[a_{1}, \ldots, a_{k}\right]=G\left[G_{1}\left[a_{1}, \ldots, a_{k}\right], \ldots, G_{p}\left[a_{1}, \ldots, a_{k}\right]\right],
$$

where $G \in G R\left(\Sigma, X_{p}\right), G_{i} \in G R\left(\Sigma, X_{k}\right)$ and $a_{i} \in M_{\alpha_{i}, \beta_{i}}, 1 \leq i \leq k$.

Let $\mathbf{M}=(M, D, \mu)$ be a $\Sigma$-graphoid. A sub- $\Sigma$-graphoid of $\mathbf{M}$ is a subset $S \subseteq M$ such that

$\left.s g_{1}\right) D \cup \mu(\Sigma) \subseteq S$,

$\left.s g_{2}\right) S$ is closed under the o- and the $\square$-operation.

Thus $S$ becomes a $\Sigma$-graphoid with operations the restriction on $S$ of the corresponding operations of $M$.

Lemma 1. Let $S$ be a sub- $\Sigma$-graphoid of $\mathbf{M}=(M, D, \mu)$. Then

$$
a_{1}, \ldots, a_{p} \in S \text { and } G \in G R\left(\Sigma, X_{p}\right) \text { implies } G\left[a_{1}, \ldots, a_{p}\right] \in S \text {. }
$$

Proof. By induction on the size of $G$. The assertion is true if $G \in \Sigma \cup X_{p} \cup D$. Next let $G$ be a graph of size $>1$; then either $G=G_{1} \circ G_{2}$ or $G=G_{1} \square G_{2}$. In the first case we have

$$
G\left[a_{1}, \ldots, a_{p}\right]=\left(G_{1} \circ G_{2}\right)\left(a_{1}, \ldots, a_{p}\right)=G_{1}\left(a_{1}, \ldots, a_{p}\right) \circ G_{2}\left(a_{1}, \ldots, a_{p}\right) \in S,
$$

because $G_{1}\left(a_{1}, \ldots, a_{p}\right), G_{2}\left(a_{1}, \ldots, a_{p}\right)$ belong by induction to $S$, and $S$ is a sub- $\Sigma$ graphoid of $\mathbf{M}$. The case $G=G_{1} \square G_{2}$ is treated in a similar way.

Let $\mathbf{M}=(M, D, \mu)$ be a $\Sigma$-graphoid and $A \subseteq M$. The sub- $\Sigma$-graphoid of $\mathbf{M}$ generated by $A$ is the smallest sub- $\Sigma$-graphoid of $\mathbf{M}$ containing $A$. It is denoted by $\langle A\rangle_{\mathrm{M}}$. Next result gives us information about the form of the elements of $<A>_{\mathrm{M}}$.

\section{Proposition 4. It holds}

$$
<A>_{\mathbf{M}}=\left\{G\left[a_{1}, \ldots, a_{p}\right] \mid G \in G R\left(\Sigma, X_{p}\right), a_{1}, \ldots, a_{p} \in A \text { and } p \geq 0\right\} .
$$

Proof. We consider the doubly ranked set $\tilde{A}=\left(A_{m, n}\right)$, where

$$
\tilde{A}_{m, n}=\left\{G\left[a_{1}, \ldots, a_{p}\right] \mid G \in G R_{m, n}\left(\Sigma, X_{p}\right), a_{1}, \ldots, a_{p} \in A \text { and } p \geq 0\right\} .
$$

By construction $D \cup \mu(\Sigma) \subseteq \tilde{A}$. On the other hand, let

$$
G\left[a_{1}, \ldots, a_{p}\right] \in \tilde{A}_{m, n} \text { and } G^{\prime}\left[a_{1}^{\prime}, \ldots, a_{q}^{\prime}\right] \in \tilde{A}_{n, k}
$$

with $G \in G R_{m, n}\left(\Sigma, X_{p}\right), G^{\prime} \in G R_{n, k}\left(\Sigma, X_{q}\right)$, respectively. We set

$$
H=G \circ\left(G^{\prime}\left[x_{p+1} / x_{1}, \ldots, x_{p+q} / x_{q}\right]\right) \in G R_{m, k}\left(\Sigma, X_{p+q}\right)
$$

then

$$
G\left[a_{1}, \ldots, a_{p}\right] \circ G^{\prime}\left[a_{1}^{\prime}, \ldots, a_{q}^{\prime}\right]=H\left[a_{1}, \ldots, a_{p}, a_{1}^{\prime}, \ldots, a_{q}^{\prime}\right] \in \tilde{A}_{m, k}
$$


and thus $\tilde{A}$ is closed under o-product. Closure under $\square$-product can be proved analogously. Therefore, $\tilde{A}$ is a sub- $\Sigma$-graphoid of $\mathbf{M}$ including $A$. Next let $S$ be a sub-graphoid of $\mathbf{M}$, such that $A \subseteq S$, according to the previous proposition

$$
a_{1}, \ldots, a_{p} \in A \text { and } G \in G R\left(\Sigma, X_{p}\right)
$$

implies $G\left[a_{1}, \ldots, a_{p}\right] \in S$, i.e., $\tilde{A} \subseteq S$. We deduce that $\tilde{A}=<A>$ M.

\section{Graph congruences}

A notion of great importance in graph theory is that of a congruence. Actually there are two kinds of graph congruence: the ordinary and the strong graph congruences which correspond respectively to the ordinary and the fully stable tree congruences (cf. [12]).

Let $\mathbf{M}=(M, D, \mu)$ be a $\Sigma$-graphoid and assume that $\sim_{m, n}$ is an equivalence on $M_{m, n}(m, n \geq 0)$ compatible with o- and $\square$-product:

$$
\begin{array}{cc}
a \sim_{m, n} a^{\prime} \text { and } b \sim_{n, k} b^{\prime}, \quad \text { implies } & a \circ b \sim_{m, k} a^{\prime} \circ b^{\prime}, \\
a \sim_{m, n} a^{\prime} \text { and } b \sim_{r, s} b^{\prime}, \quad \text { implies } \quad a \square b \sim_{m+r, n+s} a^{\prime} \square b^{\prime} .
\end{array}
$$

Then we say that $\sim=\left(\sim_{m, n}\right)$ is a congruence on $\mathbf{M}$.

The quotient sets $\left(M_{m, n} / \sim_{m, n}\right)$ are organized into a semi-magmoid $M / \sim$ by defining the corresponding operations in the natural way

$$
[a] \diamond[b]=[a \diamond b], \quad \diamond=\circ, \square,
$$

where $[a]$ stands for the $\sim$-class of the element $a$. Actually $M / \sim$ becomes a $\Sigma$-graphoid through the set

$$
D / \sim=\left\{\left[e_{0}\right],[e],\left[i_{12}\right],\left[i_{10}\right],\left[i_{21}\right],\left[i_{01}\right],[\pi]\right\}
$$

and the function

$$
\mu / \sim: \Sigma \rightarrow M / \sim, \quad(\mu / \sim)(\sigma)=[\mu(\sigma)], \quad \sigma \in \Sigma .
$$

We use the notation $\mathbf{M} / \sim$ for the so obtained $\Sigma$-graphoid. Clearly the function sending every element $a$ of $M$ into $[a]$ is a $\Sigma$-graphoid morphism from $\mathbf{M}$ to $\mathbf{M} / \sim$.

Congruences can be characterized through the substitution operation.

Proposition 5. A family of equivalences $\sim=\left(\sim_{m, n}\right)$ is a congruence on $\mathbf{M}=$ $(M, D, \mu)$ if and only if $a_{1} \sim a_{1}^{\prime}, \ldots, a_{p} \sim a_{p}^{\prime}$ and $G \in G R\left(\Sigma, X_{p}\right)$ implies

$$
G\left[a_{1}, \ldots, a_{p}\right] \sim G\left[a_{1}^{\prime}, \ldots, a_{p}^{\prime}\right] .
$$

Proof. $(\Rightarrow)$ We establish the announced implication by using induction on the size of the graph $G$. Clearly we have nothing to prove if $G \in \Sigma \cup X_{p} \cup D$. Now any 
graph $G$ of positive size can be written either as $G=G_{1} \circ G_{2}$ or $G=G_{1} \square G_{2}$ with $\operatorname{size}\left(G_{1}\right)$, size $\left(G_{2}\right)<\operatorname{size}(G)$. Then

$$
\begin{aligned}
G\left[a_{1}, \ldots, a_{p}\right] & =\left(G_{1} \circ G_{2}\right)\left[a_{1}, \ldots, a_{p}\right] \\
& =G_{1}\left[a_{1}, \ldots, a_{p}\right] \circ G_{2}\left[a_{1}, \ldots, a_{p}\right] \sim G_{1}\left[a_{1}^{\prime}, \ldots, a_{p}^{\prime}\right] \circ G_{2}\left[a_{1}^{\prime}, \ldots, a_{p}^{\prime}\right] \\
& =G\left[a_{1}^{\prime}, \ldots, a_{p}^{\prime}\right] .
\end{aligned}
$$

The $\square$-case is treated analogously.

$(\Leftarrow)$ The converse is easy to prove: we only have to take $G=\xi_{1} \diamond \xi_{2}$ with $\diamond=\circ, \square$.

Corollary 1. The equivalence $\sim$ on $G R(\Sigma, X)$ is a congruence if for all graphs $G_{i}, G_{i}^{\prime} \in G R(\Sigma, X), 1 \leq i \leq p$, and $G \in G R\left(\Sigma, X_{p}\right)$ we have

$$
G_{1} \sim G_{1}^{\prime}, \ldots, G_{p} \sim G_{p}^{\prime} \quad \text { implies } \quad G\left[G_{1}, \ldots, G_{p}\right] \sim G\left[G_{1}^{\prime}, \ldots, G_{p}^{\prime}\right] .
$$

Congruences on $G R(\Sigma, X)$ can be characterized through frame action.

Proposition 6. The equivalence $\sim=\left(\sim_{m, n}\right)$ is a congruence on $G R(\Sigma, X)$ if and only if for all $G, G^{\prime} \in G R_{m, n}(\Sigma, X)$ and all frames $F \in F R_{m, n}^{r, s}(\Sigma, X)$ we have

$$
G \sim_{m, n} G^{\prime} \text { implies } F \cdot G \sim_{r, s} F \cdot G^{\prime} .
$$

Proof. $(\Rightarrow)$ One direction comes immediately from the previous corollary:

$$
F \cdot G=F\left[G / \xi_{m, n}\right] \sim_{r, s} F\left[G^{\prime} / \xi_{m, n}\right]=F \cdot G^{\prime} .
$$

$(\Leftarrow)$ For the converse, let $G_{i}, G_{i}^{\prime} \in G R_{r_{i}, s_{i}}(\Sigma, X)$, and $G \in G R_{r, s}\left(\Sigma, X_{p}\right)$ with $\operatorname{rank}\left(x_{i}\right)=\left(r_{i}, s_{i}\right), 1 \leq i \leq p$. We may assume that the occurrences of the same variable $x_{i}$ into $G$ can be linearly ordered. For this we only have to decompose $G$ into a normal form as in Theorem 1:

$$
\begin{aligned}
G & =\Pi_{\alpha} \circ\left(I_{p_{1}, q_{1}} \square \cdots \square I_{p_{t}, q_{t}}\right) \circ \Pi_{\beta} \\
& \circ\left(E_{s} \square B F\left(x_{1}^{1}\right) \square \cdots \square B F\left(x_{1}^{\mu_{1}}\right) \square \cdots \square B F\left(x_{p}^{1}\right) \square \cdots \square B F\left(x_{p}^{\mu_{p}}\right)\right. \\
& \left.\square B F\left(\sigma_{1}^{1}\right) \square \cdots \square B F\left(\sigma_{1}^{\lambda_{1}}\right) \square \cdots \square B F\left(\sigma_{u}^{1}\right) \square \cdots \square B F\left(\sigma_{u}^{\lambda_{u}}\right)\right)
\end{aligned}
$$

where $x_{i}^{1}=\cdots=x_{i}^{\mu_{i}}=x_{i}(1 \leq i \leq p)$ and $\sigma_{j}^{1}=\cdots=\sigma_{j}^{\lambda_{j}}=\sigma_{j}, \sigma_{j} \in \Sigma$.

We introduce the frames

$$
\begin{aligned}
F_{i}^{(j)}=G\left[G_{1}^{\prime} / x_{1}, \ldots, G_{i-1}^{\prime} / x_{i-1}, G_{i}^{\prime} / x_{i}^{1}, \ldots, G_{i}^{\prime} / x_{i}^{j-1}, \xi_{r_{i}, s_{i}} / x_{i}^{j}\right. \\
\left.G_{i} / x_{i}^{j+1}, \ldots, G_{i} / x_{i}^{\lambda_{i}}, G_{i+1} / x_{i+1}, \ldots, G_{p} / x_{p}\right]
\end{aligned}
$$

then we have

$$
\begin{aligned}
G\left[G_{1}, \ldots, G_{p}\right] & =F_{1}^{1} \cdot G_{1} \sim_{r_{1}, s_{1}} F_{1}^{1} \cdot G_{1}^{\prime} \\
& =F_{1}^{2} \cdot G_{1} \sim_{r_{1}, s_{1}} F_{1}^{2} \cdot G_{1}^{\prime} \sim_{r_{1}, s_{1}} \cdots \sim_{r_{1}, s_{1}} F_{1}^{\lambda_{1}} \cdot G_{1} \sim_{r_{1}, s_{1}} F_{1}^{\lambda_{1}} \cdot G_{1}^{\prime} \\
& =G\left[G_{1}^{\prime}, G_{2}, \ldots, G_{p}\right] \sim \cdots \sim G\left[G_{1}^{\prime}, G_{2}^{\prime}, \ldots, G_{p}^{\prime}\right]
\end{aligned}
$$

and so, according to Corollary 1 the equivalence $\sim$ is a congruence. 
This result allows us to characterize the congruence generated by a relation on $G R(\Sigma, X)$.

Let $R \subseteq G R(\Sigma, X) \times G R(\Sigma, X)$, i.e., $R_{m, n} \subseteq G R_{m, n}(\Sigma, X) \times G R_{m, n}(\Sigma, X)$ for all $m, n \geq 0$. For all $G, G^{\prime} \in G R_{\alpha, \beta}(\Sigma, X)$ we set $G \sim_{R, \alpha, \beta} G^{\prime}$ if there exist $\left(H, H^{\prime}\right) \in R_{m, n}$ and $F \in F R_{m, n}^{\alpha, \beta}(\Sigma, X)$ so that

$$
G=F \cdot H, G^{\prime}=F \cdot H^{\prime} \text { or } G=F \cdot H^{\prime}, G^{\prime}=F \cdot H .
$$

The relation $\sim_{R}^{*}$ is clearly an equivalence relation on $G R(\Sigma, X)$ which by construction contains $R$. To show that $\sim_{R}^{*}$ is a congruence it suffices to show that

$$
G \sim_{R, m, n} G^{\prime} \text { and } F \in F R_{m, n}^{\alpha, \beta}(\Sigma, X) \text {, implies } F \cdot G \sim_{R, \alpha, \beta} F \cdot G^{\prime} .
$$

Indeed there exist a pair $\left(H, H^{\prime}\right) \in R_{r, s}$ and $F^{\prime} \in F R_{r, s}^{m, n}(\Sigma, X)$ so that either

$$
G=F^{\prime} \cdot H, G^{\prime}=F^{\prime} \cdot H^{\prime} \text { or } G=F^{\prime} \cdot H^{\prime}, G^{\prime}=F^{\prime} \cdot H .
$$

Hence either,

$$
F \cdot G=\left(F \cdot F^{\prime}\right) \cdot H, F \cdot G^{\prime}=\left(F \cdot F^{\prime}\right) \cdot H^{\prime}
$$

or

$$
F \cdot G=\left(F \cdot F^{\prime}\right) \cdot H^{\prime}, F \cdot G^{\prime}=\left(F \cdot F^{\prime}\right) \cdot H .
$$

By the definition of $\sim_{R}$ :

$$
F \cdot G \sim_{R, \alpha, \beta} F \cdot G^{\prime}
$$

as wanted.

Furthermore, let $\sim$ be a congruence on $G R(\Sigma, X)$ such that $R \subseteq \sim$. Since $\sim$ is reflexive and transitive, in order to show that $\sim_{R}^{*} \subseteq \sim$ it suffices to show the inclusion $\sim_{R} \subseteq \sim$. For this let $G \sim_{R, \alpha, \beta} G^{\prime}$ then for some $\left(H, H^{\prime}\right) \in R_{m, n}$ and $F \in F R_{m, n}^{\alpha, \beta}(\Sigma, X)$, we have either $G=F \cdot H, G^{\prime}=F \cdot H^{\prime}$ or $G=F \cdot H^{\prime}$, $G^{\prime}=F \cdot H$. But, according to Proposition 6

$$
H \sim_{m, n} H^{\prime} \quad \text { implies } \quad G=F \cdot H \sim_{\alpha, \beta} F \cdot H^{\prime}=G^{\prime} \quad \text { implies } \quad G \sim_{\alpha, \beta} G^{\prime} .
$$

In other words $\sim_{R}^{*}$ is the smallest congruence on $G R(\Sigma, X)$ containing $R$.

We summarize.

Theorem 4. Given $R \subseteq G R(\Sigma, X) \times G R(\Sigma, X), \sim_{R}^{*}$ is the congruence generated by $R$.

\section{$5 \quad$ Strong Graph Congruences}

Let $X=\left\{x_{1}, x_{2}, \ldots\right\}$, with $\operatorname{rank}\left(x_{i}\right)=\left(\alpha_{i}, \beta_{i}\right), i \geq 1$. An equivalence $\sim$ on $G R(\Sigma, X)$ is said to be a strong congruence if for all $p \geq 1, G, G^{\prime}, G_{i}, G_{i}^{\prime} \in$ $G R\left(\Sigma, X_{p}\right), 1 \leq i \leq p$, it holds

$$
G \sim G^{\prime} \text { and } G_{1} \sim G_{1}^{\prime}, \ldots, G_{p} \sim G_{p}^{\prime} \quad \text { implies } \quad G\left[G_{1}, \ldots, G_{p}\right] \sim G^{\prime}\left[G_{1}^{\prime}, \ldots, G_{p}^{\prime}\right] .
$$


Since the intersection of any family of such congruences has the same property we may speak of the strong congruence generated by a relation $\mathcal{E} \subseteq G R(\Sigma, X) \times$ $G R(\Sigma, X)$. It is the intersection of all strong congruences including $\mathcal{E}$ and is denoted by $<\mathcal{E}>$.

In order to get a more treatable form of $\langle\mathcal{E}\rangle$ we introduce the $p$-ranked symbols

$$
\int_{x_{1} \quad \ldots}^{\varphi_{p}^{m, n}} \operatorname{rank}\left(x_{i}\right)=\left(m_{i}, n_{i}\right), 1 \leq i \leq p \quad(m, n, p \geq 1)
$$

and we consider the set $\operatorname{REDEX}(\Sigma)_{m, n}^{\alpha, \beta}$ of all pairs $\pi=\left(F, \phi_{p}^{m, n}\left(G_{1}, \ldots, G_{p}\right)\right)$, where $F \in F R_{m, n}^{\alpha, \beta}\left(\Sigma, X_{p}\right)$ and $G_{i} \in G R_{m_{i}, n_{i}}\left(\Sigma, X_{p}\right), 1 \leq i \leq p$. For every $\pi \in$ $R E D E X(\Sigma)_{m, n}^{\alpha, \beta}$ and $\pi^{\prime} \in R E D E X(\Sigma)_{r, s}^{m, n}$ :

$$
\pi=\left(F, \varphi_{p}^{m, n}\left(G_{1}, \ldots, G_{p}\right)\right), \quad \pi^{\prime}=\left(F^{\prime}, \varphi_{p}^{r, s}\left(G_{1}^{\prime}, \ldots, G_{p}^{\prime}\right)\right)
$$

we define the product

$$
\pi \cdot \pi^{\prime}=\left(F \cdot F^{\prime}\left[G_{1} / x_{1}, \ldots, G_{p} / x_{p}\right], \varphi_{p}^{r, s}\left(G_{1}^{\prime}\left[G_{1}, \ldots, G_{p}\right], \ldots, G_{p}^{\prime}\left[G_{1}, \ldots, G_{p}\right]\right)\right) .
$$

The sets $R E D E X(\Sigma)_{m, n}^{\alpha, \beta}$ are organized into a category whose object set is $\mathbb{N} \times \mathbb{N}$ and whose composition is given by the above formula. The identity element in $R E D E X(\Sigma)_{m, n}^{m, n}$ is the pair $\varepsilon^{m, n}=\left(\xi_{m, n}, \varphi_{p}^{m, n}\left(x_{1}, \ldots, x_{p}\right)\right)$. There result actions

$$
R E D E X(\Sigma)_{m, n}^{\alpha, \beta} \times G R_{m, n}\left(\Sigma, X_{p}\right) \rightarrow G R_{\alpha, \beta}\left(\Sigma, X_{p}\right), \quad \alpha, \beta, m, n \geq 0,
$$

defined as follows: for $\pi=\left(F, \varphi_{p}^{m, n}\left(G_{1}, \ldots, G_{p}\right)\right)$ and $G \in G R_{m, n}\left(\Sigma, X_{p}\right)$ we set

$$
\text { (act) } \quad \pi \cdot G=F \cdot G\left[G_{1}, \ldots, G_{p}\right] .
$$

The formulas $\left(\pi \cdot \pi^{\prime}\right) \cdot G=\pi \cdot\left(\pi^{\prime} \cdot G\right), \varepsilon^{m, n} \cdot G=G$ folow.

Proposition 7. The equivalence $\sim=\left(\sim_{m, n}\right)$ in $G R\left(\Sigma, X_{p}\right)$ is a strong congruence iff it is compatible with the above actions.

Proof. $(\Rightarrow)$ Suppose that $G \sim_{m, n} G^{\prime}$ and

$$
\pi=\left(F, \varphi_{p}^{m, n}\left(G_{1}, \ldots, G_{p}\right)\right) \in R E D E X(\Sigma)_{m, n}^{\alpha, \beta}
$$

then we have $G\left[G_{1}, \ldots, G_{p}\right] \sim_{m, n} G^{\prime}\left[G_{1}, \ldots, G_{p}\right]$ and thus by Proposition 6 we get

$$
F \cdot G\left[G_{1}, \ldots, G_{p}\right] \sim_{\alpha, \beta} F \cdot G^{\prime}\left[G_{1}, \ldots, G_{p}\right]
$$

that is $\pi \cdot G \sim_{\alpha, \beta} \pi \cdot G^{\prime}$.

$(\Leftarrow)$ Conversely, assume that

$$
G \sim_{m, n} G^{\prime} \quad \text { and } \quad G_{i} \sim_{m_{i}, n_{i}} G_{i}^{\prime}, \quad 1 \leq i \leq p
$$


and choose

$$
\pi=\left(F, \varphi_{p}^{m, n}\left(x_{1}, \ldots, x_{p}\right)\right), \quad F \in F R_{m, n}^{\alpha, \beta}\left(\Sigma, X_{p}\right)
$$

then we have

$$
\pi \cdot G \sim_{\alpha, \beta} \pi \cdot G^{\prime} \text {, i.e., } F \cdot G \sim_{\alpha, \beta} F \cdot G^{\prime}
$$

and thus $\sim$ is a congruence (see Proposition 6). Consequently, we have

$$
G\left[G_{1}, \ldots, G_{p}\right] \sim_{m, n} G\left[G_{1}^{\prime}, \ldots, G_{p}^{\prime}\right]
$$

choosing this time $\pi=\left(\xi_{m, n}, \varphi_{p}^{m, n}\left(G_{1}^{\prime}, \ldots, G_{p}^{\prime}\right)\right)$ we obtain $\pi \cdot G \sim_{m, n} \pi \cdot G^{\prime}$ or

$$
G\left[G_{1}^{\prime}, \ldots, G_{p}^{\prime}\right] \sim_{m, n} G^{\prime}\left[G_{1}^{\prime}, \ldots, G_{p}^{\prime}\right] .
$$

Combining $(\sigma)$ and $(\tau)$ above we find

$$
G\left[G_{1}, \ldots, G_{p}\right] \sim_{m, n} G^{\prime}\left[G_{1}^{\prime}, \ldots, G_{p}^{\prime}\right]
$$

i.e., $\sim$ is a strong congruence.

We are now ready to describe the strong congruence generated by a set $\mathcal{E} \subseteq$ $G R(\Sigma, X) \times G R(\Sigma, X)$. For $H, H^{\prime} \in G R_{\alpha, \beta}\left(\Sigma, X_{p}\right)$, we write $H \overleftrightarrow{\mathcal{E}} H^{\prime}$ if there exist $\pi \in R E D E X(\Sigma)_{m, n}^{\alpha, \beta}$ and $\left(G, G^{\prime}\right) \in \mathcal{E}, G, G^{\prime} \in G R_{m, n}\left(\Sigma, X_{p}\right)$ so that either

$$
H=\pi \cdot G, \quad H^{\prime}=\pi \cdot G^{\prime} \quad \text { or } \quad H=\pi \cdot G^{\prime}, \quad H^{\prime}=\pi \cdot G .
$$

We denote by $\underset{\mathcal{E}}{\stackrel{*}{\leftrightarrow}}$, the reflexive and transitive closure of $\underset{\mathcal{E}}{\overleftrightarrow{ }}$, i.e., $H \underset{\mathcal{E}}{\stackrel{*}{\leftrightarrow}} H^{\prime}$ if

$$
H=H_{0} \underset{\mathcal{E}}{\overleftrightarrow{\mathcal{E}}} H_{1} \underset{\mathcal{E}}{\overleftrightarrow{\mathcal{E}}} H_{k-1} \underset{\mathcal{E}}{\overleftrightarrow{\mathcal{E}}} H_{k}=H^{\prime}
$$

for some $k \geq 0$. The $\underset{\mathcal{E}}{\stackrel{*}{\leftrightarrow}}$ is by construction an equivalence relation.

If $H \underset{\mathcal{E}}{\leftrightarrow} H^{\prime}$, then by definition,

$$
H=\pi \cdot G, \quad H^{\prime}=\pi \cdot G^{\prime} \quad \text { or } \quad H=\pi \cdot G^{\prime}, \quad H^{\prime}=\pi \cdot G,
$$

for some $\pi \in R E D E X(\Sigma)_{m, n}^{\alpha, \beta}$ and $\left(G, G^{\prime}\right) \in \mathcal{E}$ and so for every $\bar{\pi} \in R E D E X(\Sigma)_{\alpha, \beta}^{\gamma, \delta}$ we shall have

$$
\bar{\pi} \cdot H=(\bar{\pi} \cdot \pi) \cdot G, \quad \bar{\pi} \cdot H^{\prime}=(\bar{\pi} \cdot \pi) \cdot G^{\prime}
$$

or

$$
\bar{\pi} \cdot H=(\bar{\pi} \cdot \pi) \cdot G^{\prime}, \quad \bar{\pi} \cdot H^{\prime}=(\bar{\pi} \cdot \pi) \cdot H
$$

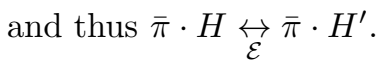

It turns out that $\underset{\mathcal{E}}{\stackrel{*}{\leftrightarrow}}$ is compatible with $(a c t)$ and so it is a strong congruence by virtue of the previous proposition. Now if $\sim$ is a strong congruence on $\operatorname{GR}(\Sigma, X)$ 


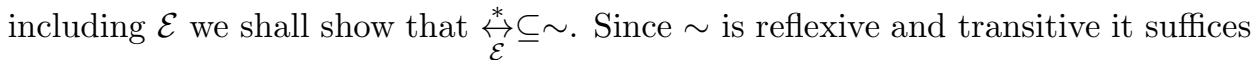
to show that $\overleftrightarrow{\mathcal{E}} \subseteq \sim$. For this let $H \underset{\mathcal{E}}{\leftrightarrow} H^{\prime}$, i.e.,

$$
H=\pi \cdot G, \quad H^{\prime}=\pi \cdot G^{\prime} \quad \text { or } \quad H=\pi \cdot G^{\prime}, \quad H^{\prime}=\pi \cdot G,
$$

for $\pi \in R E D E X(\Sigma)_{m, n}^{\alpha, \beta}$ and $\left(G, G^{\prime}\right) \in \mathcal{E}$. As $\mathcal{E} \subseteq \sim$ we shall have $G \sim_{m, n} G^{\prime}$ and so $\pi \cdot G \sim_{\alpha, \beta} \pi \cdot G^{\prime}$. Hence, $H \sim_{\alpha, \beta} H^{\prime}$. In other words, $\underset{\mathcal{E}}{\stackrel{*}{*}}$ is the smallest strong congruence including $\mathcal{E}$. We state

Theorem 5. Given a relation $\mathcal{E} \subseteq G R(\Sigma, X) \times G R(\Sigma, X)$, the $\underset{\mathcal{E}}{\stackrel{*}{*}}$ is the strong congruence generated by $\mathcal{E}$.

\section{Pattern Congruences}

In this section we discuss congruences on patterns in connection with congruences on graphs.

Pattern substitution is obtained in a natural way. Let $X=\left\{x_{1}, x_{2}, \ldots\right\}$, $\operatorname{rank}\left(x_{i}\right)=\left(m_{i}, n_{i}\right), i \geq 1$ and set $X_{k}=\left\{x_{1}, \ldots, x_{k}\right\}$. For $p \in \operatorname{mag}_{\alpha, \beta}\left(\Sigma \cup X_{k}\right)$ and $p_{i} \in \operatorname{mag}_{m_{i}, n_{i}}(\Sigma), 1 \leq i \leq k$, the pattern $p\left[p_{1}, \ldots, p_{k}\right]$ is by definition the image of $p$ via the magmoid morphism $\operatorname{mag}\left(\Sigma \cup X_{k}\right) \rightarrow \operatorname{mag}(\Sigma)$ defined by the assignments

$$
x_{1} \mapsto p_{1}, \ldots, x_{k} \mapsto p_{k}, \quad \sigma \mapsto \sigma(\sigma \in \Sigma) .
$$

The set $\operatorname{Fr}(\Sigma)_{m, n}^{\alpha, \beta}$ of pattern frames is the subset of $\operatorname{mag}_{\alpha, \beta}\left(\Sigma \cup \xi_{m, n}\right)$ consisting of all patterns with just one occurrence of the symbol $\xi_{m, n}, \operatorname{rank}\left(\xi_{m, n}\right)=(m, n)$. Again $\operatorname{Fr}(\Sigma)_{m, n}^{\alpha, \beta}$ acts on $\operatorname{mag}_{m, n}(\Sigma)$ via substitution

$$
f \cdot p=f\left[p / \xi_{m, n}\right] \quad \text { for } f \in \operatorname{Fr}(\Sigma)_{m, n}^{\alpha, \beta}, \quad p \in \operatorname{mag}_{m, n}(\Sigma) .
$$

Given an equivalence relation $\mathcal{S}_{m, n}$ on $\operatorname{mag}_{m, n}(\Sigma)$, we say that $\mathcal{S}=\left(\mathcal{S}_{m, n}\right)$ is a congruence on $\operatorname{mag}(\Sigma)$ whenever we have compatibility with horizontal and vertical pattern concatenation, i.e.,

$$
p_{1} \equiv p_{1}^{\prime}\left(\mathcal{S}_{m, n}\right) \text { and } p_{2} \equiv p_{2}^{\prime}\left(\mathcal{S}_{n, k}\right) \text { imply } p_{1} p_{2} \equiv p_{1}^{\prime} p_{2}^{\prime}\left(\mathcal{S}_{m, k}\right)
$$

and

$$
p_{1} \equiv p_{1}^{\prime}\left(\mathcal{S}_{m, n}\right) \text { and } p_{2} \equiv p_{2}^{\prime}\left(\mathcal{S}_{r, s}\right) \text { imply }\left(\begin{array}{l}
p_{1} \\
p_{2}
\end{array}\right) \equiv\left(\begin{array}{l}
p_{1}^{\prime} \\
p_{2}^{\prime}
\end{array}\right)\left(\mathcal{S}_{m+r, n+s}\right) \text {. }
$$

Of course the quotients $\operatorname{mag}_{m, n}(\Sigma) / \mathcal{S}_{m, n}$ are organized, in the obvious way, into a magmoid denoted by $\operatorname{mag}(\Sigma) / \mathcal{S}$.

Proposition 8. Given an equivalence $\mathcal{S} \subseteq \operatorname{mag}(\Sigma) \times \operatorname{mag}(\Sigma)$ next conditions are equivalent: 
i) $\mathcal{S}$ is a congruence;

ii) $\mathcal{S}$ is compatible with frame action, i.e.,

$$
p \equiv p^{\prime}\left(\mathcal{S}_{m, n}\right) \text { and } f \in F r(\Sigma)_{m, n}^{\alpha, \beta} \text { imply } f \cdot p \equiv f \cdot p^{\prime}\left(\mathcal{S}_{\alpha, \beta}\right) ;
$$

iii) $\mathcal{S}$ is compatible with substitution, i.e.,

$$
p_{i} \equiv p_{i}^{\prime}\left(\mathcal{S}_{m_{i}, n_{i}}\right), 1 \leq i \leq k, \text { imply } p\left[p_{1}, \ldots, p_{k}\right] \equiv p\left[p_{1}^{\prime}, \ldots, p_{k}^{\prime}\right],
$$

for all $p \in \operatorname{mag}_{m, n}\left(\Sigma \cup X_{k}\right)$.

We now return to the standard magmoid morphism

$$
\operatorname{val}_{\Sigma}: \operatorname{mag}(\Sigma \cup \bar{D}) \rightarrow G R(\Sigma)
$$

whose kernel, denoted by $\sim_{\Sigma}$,

$$
p_{1} \sim_{\Sigma} p_{2} \text { if } \operatorname{val}_{\Sigma}\left(p_{1}\right)=\operatorname{val}_{\Sigma}\left(p_{2}\right)
$$

coincides with the congruence generated by the set of relations $(E)$ (see Subsection 2.2). Given any pattern congruence $\mathcal{S} \subseteq \operatorname{mag}(\Sigma \cup \bar{D}) \times \operatorname{mag}(\Sigma \cup \bar{D})$ containing $(E)$, its projection $\operatorname{val}_{\Sigma}(\mathcal{S})$ defined by

$$
G_{1} \equiv G_{2}\left(\operatorname{val}_{\Sigma}(\mathcal{S})\right) \quad \text { if } \quad G_{i}=\operatorname{val}_{\Sigma}\left(p_{i}\right), 1 \leq i \leq 2, p_{1} \equiv p_{2}(\mathcal{S})
$$

is a graph congruence.

Conversely, for any congruence $\mathcal{R} \subseteq G R(\Sigma) \times G R(\Sigma)$ its inverse image $v_{a l}^{-1}(\mathcal{R})$ defined by

$$
p_{1} \equiv p_{2}\left(\operatorname{val}_{\Sigma}^{-1}(\mathcal{R})\right) \quad \text { iff } \quad \operatorname{val}_{\Sigma}\left(p_{1}\right) \equiv \operatorname{val}_{\Sigma}\left(p_{2}\right)(\mathcal{R})
$$

is a congruence on $\operatorname{mag}(\Sigma \cup \bar{D})$ containing $(E)$. Therefore

Proposition 9. The mappings

$$
\mathcal{S} \mapsto \operatorname{val}_{\Sigma}(\mathcal{S}) \text { and } \mathcal{R} \mapsto \operatorname{val}_{\Sigma}^{-1}(\mathcal{R})
$$

establish a bijection between the congruences on $G R(\Sigma)$ and the congruences on $\operatorname{mag}(\Sigma \cup \bar{D})$ including $(E)$.

By working as in Section 4 we can show that the congruence generated by the relation $S \subseteq \operatorname{mag}(\Sigma) \times \operatorname{mag}(\Sigma)$ is the reflexive and transitive closure of $\sim_{S}$ with

$$
p_{1} \sim_{S} p_{2} \quad \text { iff } \quad p_{i}=f \cdot q_{i}, \quad(1 \leq i \leq 2),
$$

$f \in \operatorname{Fr}(\Sigma)$ and either $\left(q_{1}, q_{2}\right) \in S$ or $\left(q_{2}, q_{1}\right) \in S$.

We have the next remarkable result. 
Proposition 10. It holds

$$
\operatorname{val}_{\Sigma}\left(\stackrel{*}{\sim}_{S \cup(E)}\right)=\stackrel{*}{\sim}_{\operatorname{val}_{\Sigma}(S)}, \quad S \subseteq \operatorname{mag}(\Sigma \cup \bar{D}) \times \operatorname{mag}(\Sigma \cup \bar{D})
$$

that is the graph congruence generated by $\operatorname{val}_{\Sigma}(S)$ coincides with the projection, via $v l_{\Sigma}$, of the congruence generated by $S \cup(E)$.

Proof. By construction $v a l_{\Sigma}\left(\stackrel{*}{\sim}_{S \cup(E)}\right)$ is a congruence on $G R(\Sigma)$ containing $\operatorname{val}_{\Sigma}(S)$. Now, if $\mathcal{R}$ is a congruence with $\mathcal{R} \supseteq \operatorname{val}_{\Sigma}(S)$, then $\operatorname{val}_{\Sigma}^{-1}(\mathcal{R})$ is a congruence on $\operatorname{mag}(\Sigma \cup \bar{D})$ containing $S$ and so

$$
\operatorname{val}_{\Sigma}^{-1}(\mathcal{R}) \supseteq \stackrel{*}{\sim}_{S \cup(E)}
$$

Projecting by val $_{\Sigma}$ we get

$$
\mathcal{R}=\operatorname{val}_{\Sigma}\left(\operatorname{val}_{\Sigma}^{-1}(\mathcal{R})\right) \supseteq \operatorname{val}_{\Sigma}\left(\stackrel{*}{\sim}_{S \cup(E)}\right),
$$

i.e., $\operatorname{val}_{\Sigma}\left(\stackrel{*}{\sim}_{S \cup(E)}\right)$ is the smallest congruence containing $\operatorname{val}_{\Sigma}(S)$. Hence the result.

Proposition 11. If $R \subseteq G R(\Sigma) \times G R(\Sigma)$ is a relation then

$$
\operatorname{val}_{\Sigma}^{-1}\left(\stackrel{*}{\sim}_{R}\right)=\stackrel{*}{\sim}_{v_{a l}}^{-1}(R) \cup(E) .
$$

Proof. By construction $\operatorname{val}_{\Sigma}^{-1}\left(\stackrel{*}{\sim}_{R}\right)$ is a congruence including $\operatorname{val}_{\Sigma}^{-1}(R) \cup(E)$ while if $\mathcal{S}$ is a congruence on $\operatorname{mag}(\Sigma \cup \bar{D})$ with

$$
\mathcal{S} \supseteq \operatorname{val}_{\Sigma}^{-1}(R) \cup(E),
$$

then its projection $\operatorname{val}_{\Sigma}(S)$ is a congruence on $G R(\Sigma)$ such that

$$
\operatorname{val}_{\Sigma}\left(\operatorname{val}_{\Sigma}^{-1}(R \cup(E))\right)=\operatorname{val}_{\Sigma}\left(\operatorname{val}_{\Sigma}^{-1}(R)\right) \cup \operatorname{val}_{\Sigma}((E))=R .
$$

Thus $\stackrel{*}{\sim}_{R} \subseteq \operatorname{val}_{\Sigma}(S)$ and so

$$
\operatorname{val}_{\Sigma}\left(\stackrel{*}{\sim}_{R}\right) \subseteq \operatorname{val}_{\Sigma}^{-1}\left(\operatorname{val}_{\Sigma}(S)\right)=S
$$

i.e., $\operatorname{val}_{\Sigma}^{-1}\left(\stackrel{*}{\sim}_{R}\right)$ is the congruence generated by $\operatorname{val}_{\Sigma}^{-1}(R) \cup(E)$, as wanted.

In the sequel we discuss strong pattern congruences. An equivalence $\mathcal{S}=\left(\mathcal{S}_{m, n}\right)$ on $\operatorname{mag}(\Sigma \cup X)$ is called a strong congruence if for every $k \geq 0$ and $p, p^{\prime} \in \operatorname{mag}_{\alpha, \beta}(\Sigma \cup$ $\left.X_{k}\right), p_{i}, p_{i}^{\prime} \in \operatorname{mag}_{m_{i}, n_{i}}(\Sigma \cup X), 1 \leq i \leq k$, we have

$$
p \equiv p^{\prime}\left(\mathcal{S}_{\alpha, \beta}\right) \quad \text { and } \quad p_{i} \equiv p_{i}^{\prime}\left(\mathcal{S}_{m_{i}, n_{i}}\right), \quad 1 \leq i \leq k
$$

imply

$$
p\left[p_{1}, \ldots, p_{k}\right] \equiv p^{\prime}\left[p_{1}^{\prime}, \ldots, p_{k}^{\prime}\right]\left(\mathcal{S}_{\alpha, \beta}\right)
$$


Here we also can achieve an action characterization. For this we introduce the set of redexes $\operatorname{Redex}(\Sigma)_{m, n}^{\alpha, \beta}$ consisting of all pairs

$$
\left(f, \varphi_{k}^{m, n}\left(p_{1}, \ldots, p_{k}\right)\right), \quad f \in \operatorname{Fr}(\Sigma)_{m, n}^{\alpha, \beta}, p_{i} \in \operatorname{mag}_{m_{i}, n_{i}}(\Sigma \cup X), 1 \leq i \leq k .
$$

where $\varphi_{k}^{m, n}$ are $k$-ranked symbols as in Section 4 .

These sets are organized into a category whose object set is $\mathbb{N} \times \mathbb{N}$ and whose composition is defined by

$$
\begin{aligned}
\left(f, \varphi_{k}^{m, n}\left(p_{1}, \ldots, p_{k}\right)\right) & \cdot\left(f^{\prime}, \varphi_{k}^{r, s}\left(p_{1}^{\prime}, \ldots, p_{k}^{\prime}\right)\right) \\
& =\left(f \cdot f^{\prime}\left[p_{1}, \ldots, p_{k}\right], \varphi_{k}^{r, s}\left(p_{1}^{\prime}\left[p_{1}, \ldots, p_{k}\right], \ldots, p_{k}^{\prime}\left[p_{1}, \ldots, p_{k}\right]\right)\right) .
\end{aligned}
$$

There results a canonical action

$$
\operatorname{Redex}(\Sigma)_{m, n}^{\alpha, \beta} \times \operatorname{mag}_{m, n}(\Sigma \cup X) \rightarrow \operatorname{mag}_{\alpha, \beta}(\Sigma \cup X)
$$

if for every $\pi=\left(f, \varphi_{k}^{m, n}\left(p_{1}, \ldots, p_{k}\right)\right)$ and $p \in \operatorname{mag}_{m, n}\left(\Sigma \cup X_{k}\right)$ we set

$$
\pi \cdot p=\left(f \cdot p\left[p_{1}, \ldots, p_{k}\right]\right)
$$

Proposition 12. The equivalence $\mathcal{S} \subseteq \operatorname{mag}(\Sigma \cup X) \times \operatorname{mag}(\Sigma \cup X)$ is a strong congruence if and only if it is compatible with the above action i.e.,

$$
p \equiv p^{\prime}\left(\mathcal{S}_{m, n}\right) \text { and } \pi \in \operatorname{Cont}(\Sigma)_{m, n}^{\alpha, \beta}
$$

implies

$$
\pi \cdot p \equiv \pi \cdot p^{\prime}\left(\mathcal{S}_{\alpha, \beta}\right)
$$

Theorem 6. The strong congruence generated by the relation

$$
S \subseteq \operatorname{mag}(\Sigma \cup X) \times \operatorname{mag}(\Sigma \cup X)
$$

is $\stackrel{*}{\leftrightarrow}_{S}$, the reflexive and transitive closure of $\leftrightarrow_{S}$ defined by

$$
p_{1} \leftrightarrow_{S} p_{i} \quad \text { iff } \quad p_{i}=\pi \cdot q_{i} \quad(1 \leq i \leq 2)
$$

with $\pi \in \operatorname{Cont}(\Sigma)$ and either $\left(q_{1}, q_{2}\right) \in S$ or $\left(q_{2}, q_{1}\right) \in S$.

Let $\operatorname{val}_{\Sigma, X}: \operatorname{mag}(\Sigma \cup \bar{D} \cup X) \rightarrow G R(\Sigma \cup X)$ be the magmoid morphism extending val $_{\Sigma}$ by setting val $_{\Sigma, X}(x)=x$, for all $x \in X$.

Theorem 7. The mapping $\mathcal{R} \mapsto \operatorname{val}_{\Sigma, X}^{-1}(\mathcal{R})$ and $S \mapsto \operatorname{val}_{\Sigma, X}(S)$ establish a bijection between the strong congruences $\mathcal{R}$ of $G R(\Sigma \cup X)$ and the strong congruences $\mathcal{S}$ on $\operatorname{mag}(\Sigma \cup \bar{D} \cup X)$ including the set $E_{X}$ obtained by adding to $(E)$ the set of relations

$$
\bar{\pi}_{m, 1}\left(\begin{array}{l}
x \\
e
\end{array}\right) \sim\left(\begin{array}{l}
e \\
x
\end{array}\right) \bar{\pi}_{n, 1}, \quad x \in X
$$

Moreover, given relations

$$
S \subseteq \operatorname{mag}(\Sigma \cup \bar{D} \cup X)^{2}, \quad R \subseteq G R(\Sigma \cup X)^{2}
$$

it holds

$$
\operatorname{val}_{\Sigma, X}^{-1}\left(\stackrel{*}{\leftrightarrow}_{R}\right)=\stackrel{*}{\leftrightarrow}_{v a l_{\Sigma, X}^{-1}(R) \cup E_{X}},
$$

and

$$
\operatorname{val}_{\Sigma, X}\left(\stackrel{*}{\leftrightarrow}_{S \cup E_{X}}\right)=\stackrel{*}{\leftrightarrow}_{v a l_{\Sigma, X}(S)} .
$$




\section{Birkhoff's Variety Theorem for Graphs}

The important Birkhoff's variety theorem is valid for graphs due to the universal characterization of $G R(\Sigma)$ displayed in Theorem 3. We must point out that most of the results of this section can be obtained by suitably adapting the proof argument of the corresponding results for trees (cf. [12]). Hence we will only present, without proofs, the main theorems in this direction. Let us begin with a classical definition.

A class $\mathcal{V}$ of $\Sigma$-graphoids forms a variety of $\Sigma$-graphoids ( $\Sigma$ - $G$-variety in short) if it is closed under isomorphism, cartesian products, sub- $\Sigma$-graphoids and quotients. Of course cartesian products are defined in the classical categorical manner.

Theorem 8. Let $\mathcal{V}$ be a $\Sigma$-G-variety. For every set $X$ the (doubly ranked) function

$$
j_{X}: X \rightarrow G R(\mathcal{V}, X), \quad j_{X}(x)=x, x \in X
$$

has the following universal property: for every $\mathbf{M} \in \mathcal{V}$ and every (doubly ranked) function $g: X \rightarrow \mathbf{M}$, there is a unique $\Sigma$-graphoid morphism $\tilde{g}: G R(\mathcal{V}, X) \rightarrow \mathbf{M}$ rendering commutative the triangle

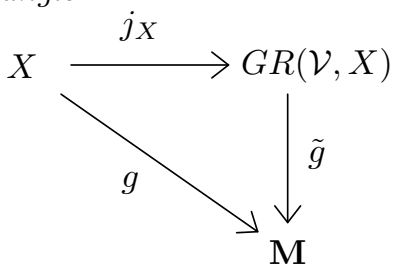

Remark 1. The previous result means that $G R(\mathcal{V}, X)$ is the free $\Sigma$-graphoid over $\mathcal{V}$ generated by $X$ and its elements can be considered as "graphs inside $\mathcal{V}$ ".

In the case that $X=\emptyset$, the $\Sigma$-graphoid $G R(\mathcal{V})=G R(\mathcal{V}, \emptyset)$ has the characteristic property: for all $\mathbf{M} \in \mathcal{V}$ there is a unique $\Sigma$-graphoid morphism $h_{\mathcal{V}, \mathbf{M}}: G R(\mathcal{V}) \rightarrow$ M.

Theorem 9. Every $\Sigma$-G-variety $\mathcal{V}$ is generated by the $\Sigma$-graphoids $G R\left(\mathcal{V}, X_{n}\right)$, $n \geq 0$, i.e.,

$$
\mathcal{V}=V\left(\left\{G R\left(\mathcal{V}, X_{n}\right) \mid n \geq 0\right\}\right) .
$$

Next we discuss equationality of $\Sigma$ - $G$-varieties.

A graph equation over the doubly ranked alphabet $\Sigma$ is just a pair $\left(G, G^{\prime}\right)$ where $G, G^{\prime} \in G R\left(\Sigma, X_{n}\right)$, for some $n \geq 0$. Frequently a graph equation $\left(G, G^{\prime}\right)$ will be denoted by $G=G^{\prime}$. We say that the equation $\left(G, G^{\prime}\right)$ is satisfied in the $\Sigma$-graphoid $\mathbf{M}$ (or that $\mathbf{M}$ is a model of $\left(G, G^{\prime}\right)$ ) whenever

$$
G\left[a_{1}, \ldots, a_{n}\right]=G^{\prime}\left[a_{1}, \ldots, a_{n}\right], \quad \text { for all } a_{1}, \ldots, a_{n} \in \mathbf{M} .
$$

This fact is denoted by $\mathbf{M} \models\left(G, G^{\prime}\right)$.

For a class $\mathcal{K}$ of $\Sigma$-graphoids, we write $\mathcal{K} \models\left(G, G^{\prime}\right)$ if $\mathbf{M} \models\left(G, G^{\prime}\right)$, for all $\mathbf{M} \in \mathcal{K}$. By $\operatorname{Mod}\left(G, G^{\prime}\right)$ we denote the class of all models of $\left(G, G^{\prime}\right)$ and for a set of equations $\mathcal{E} \subseteq G R(\Sigma, X) \times G R(\Sigma, X)$, we set

$$
\operatorname{Mod}(\mathcal{E})=\bigcap_{\left(G, G^{\prime}\right) \in \mathcal{E}} \operatorname{Mod}\left(G, G^{\prime}\right)
$$


Clearly $\operatorname{Mod}(\mathcal{E})$ is a $\Sigma$ - $G$-variety. We say that a $\Sigma$ - $G$-variety is equationally defined whenever $\mathcal{V}=\operatorname{Mod}(\mathcal{E})$ for some $\mathcal{E}$.

Given a class $\mathcal{K}$ of $\Sigma$-graphoids, $E q(\mathcal{K})$ is the set of all equations $\left(G, G^{\prime}\right)$ with $\mathcal{K} \models\left(G, G^{\prime}\right)$. It is easy to see that $E q(\mathcal{K})$ is a strong congruence on $G R(\Sigma, X)$.

Applying Theorem 8 we get that for any $\Sigma$ - $G$-variety $\mathcal{V}$ we have

$$
E q(\mathcal{V})=\sim \mathcal{V}, X
$$

Proposition 13. For every $\Sigma$-G-variety $\mathcal{V}$, it holds

$$
\mathcal{V}=\operatorname{Mod}(E q(\mathcal{V}))
$$

Consequently every $\Sigma$-G-variety is equationally defined.

Theorem 10 (Birkhoff). The correspondences

$$
\mathcal{R} \mapsto \operatorname{Mod}(\mathcal{R}), \quad \mathcal{V} \mapsto E q(\mathcal{V})
$$

define a bijection between the class of all $\Sigma$-G-varieties and the class of all strong congruences on $G R(\Sigma, X)$.

Theorem 11 (Completness). For any set $\mathcal{E}$ of equations in $G R(\Sigma, X)$, we have

$$
\operatorname{Mod}(\mathcal{E}) \models\left(G, G^{\prime}\right) \text { if and only if } G \stackrel{\leftrightarrow}{\stackrel{\mathcal{E}}{\longrightarrow}} G^{\prime},
$$

i.e., if the equation $\left(G, G^{\prime}\right)$ is satisfied by every model of $\mathcal{E}$ then we can go from $G$ to $G^{\prime}$ by means of the equations of $\mathcal{E}$ and vise versa.

\section{References}

[1] A. Arnold and M. Dauchet, Théorie des magmoides. I. RAIRO Inform. Théor. 12 (3), 235-257 (1978).

[2] A. Arnold and M. Dauchet, Théorie des magmoides. II. RAIRO Inform. Théor. 13 (2), 135-154 (1979).

[3] F. Baader and T. Nipkow, Term Rewriting and All That, Cambridge University Press, (1999).

[4] S. Bozapalidis and A. Kalampakas, An axiomatization of graphs Acta Informatica 41, 19 - 61 (2004).

[5] S. Bozapalidis and A. Kalampakas, Recognizability of graph and pattern languages. Acta Informatica 42, 553 - 581 (2006).

[6] S. Bozapalidis and A. Kalampakas, Graph Automata, Theoretical Computer Science 393, 147 - 165 (2008). 
[7] P.M. Cohn, Universal Algebra, D. Reidel Publishing, Dordrecht, Netherlands, (1981)

[8] F. Drewes, H.-J. Kreowski and A. Habel, Hyperedge Replacement, Graph Grammars. Handbook of Graph Grammars, 95-162, (1997).

[9] J. Engelfriet, Context-free graph grammars. In G. Rozenberg and A. Salomaa, eds, Handbook of Formal Languages. Vol. III: Beyond Words, chapter 3, pages 125-213. Springer, 1997.

[10] J. Engelfriet, J.J. Vereijken, Context-free graph grammars and concatenation of graphs. Acta Informatica 34, 773-803, (1997).

[11] J.W. Klop, Term rewriting systems, in S. Abramsky, D.M. Gabbay, and T.S.E. Maibaum, editors, Handbook of Logic in Computer Science, volume 2, pages 1116. Oxford University Press, 1992.

[12] W. Wechler, Universal Algebra for Computer Scientists. Grzegorz Rozenberg, Arto Salomaa, W. Brauer (Eds), Eatcs Monographs on Theoretical Computer 25, Springer-Verlag New York, (1992). 